\title{
HOW TO OPERATE PHYSICAL SHOWROOMS: SERVICE DECISION AND PRICING BASED ON PRODUCT QUALITY
}

\author{
Chunxiang Guo, Yuyang Tan* and Yue Tan
}

\begin{abstract}
This paper addresses operation strategies of the physical showroom under e-commerce and studies joint decisions of the showroom service and pricing based on product quality. First, we propose an analytical model to capture the feature of consumer purchase behaviors and examine the three operation strategies: the cooperation operation $(\mathrm{CO})$, the manufacturer operation (MO), and the e-retailer operation ( $\mathrm{RO}$ ). Then, the equilibriums in the above three strategies are obtained and analyzed based on product quality. Besides, we extend the model to the scenario of endogenous product quality. The results are as follows. First, in contrast to the conventional wisdom, we find that the optimal showroom service may decrease with product quality when the production cost coefficient is high. Second, both product quality and production cost should be considered before opening physical showrooms. When the production cost coefficient is low, the MO strategy and the RO strategy are better than the CO strategy, which means that the enterprise should open the physical showroom separately. However, when the production cost coefficient is high, the CO strategy provides the optimal showroom service and the enterprises should cooperate to balance costs and benefits. Moreover, when product quality is endogenous, our analysis reveals that the RO strategy may offer better product quality than the CO strategy when the production cost coefficient is moderate.
\end{abstract}

Mathematics Subject Classification. 90B60.

Received February 28, 2021. Accepted November 25, 2021.

\section{INTRODUCTION}

Given the convenience of product information accessibility, consumers are no longer loyal to a single channel. They are becoming sophisticated enough to switch their purchase channels based on multi-channel information comparison [4]. Meanwhile, consumers not only consider product quality, but also pay more attention to the psychological experience obtained during the purchase process. To accommodate these purchase behaviors, plenty of enterprises begin to transform from traditional retailing to omnichannel operation $[2,40]$. Particularly, the physical showroom, one of the omnichannel strategies, has proved to be effective in academia [3]. In industry, Numerous e-commerce enterprises, such as Amazon Go, and Hema by Alibaba, have opened the physical showroom to provide complementary experience services for consumers [7].

As a new retail pattern, from the perspective of enterprises, the physical showroom aims at solving the uncertainty issue of product information, simultaneously voiding conflicts between channels [19]. Previous works

Keywords. Omnichannel, operation strategy, physical showroom, product quality.

Business School, Sichuan University, Chengdu, P.R. China.

*Corresponding author: tyytanyuyang@163.com 
and practical cases point out that introduction of the physical showroom benefits enterprises because it increases online sales and improves the overall operational efficiency of the supply chain [4]. In the physical showroom, the specific measures, consisting of product assortments, advertising, and high-quality salesperson, are adopted and proved effective to improve the customer experience and achieve diversified consumption scenarios [29]. Moreover, the application of information technologies, such as self-order technology and mobile app, also thrives its prosperity $[20,30]$.

However, despite the feasibility of the physical showroom, it is still unclear how to operate the physical showroom effectively. In practice, there are three main operation strategies described as follows. The first is the manufacturer operation (MO). For example, the British furniture manufacturer Made.com has opened its first showroom in 2012, and both real furniture and 3D-printed models are exhibited. Consumers can scan the QR code to obtain detailed information about their target products ${ }^{1}$. Besides, electronics firms, such as Apple, and Huawei have also opened plenty of showrooms in recent years. The second is the e-retailer operation (RO). Well-known e-retailers, such as Warby Parker and Bonobos, encourage customers to engage in their products profoundly by opening the physical showroom [4]. The third is the cooperation operation (CO), representing vertical cooperation between the manufacturer and the e-retailer in the supply chain. For example, the physical showroom of 5G technology, jointly built by China Telecom and JD, has been officially opened in November $2019^{2}$.

From the perspective of consumers, as they cannot directly touch target products when shopping online, the physical showroom is available for them to find and inspect their target products. The physical showroom not only promotes the overall efficiency of the supply chain under e-commerce, but also leaves customers a good impression on the product quality. It's worth noting that product quality in the paper refers to all relevant aspects of a product in the consumer purchase decision [5,23]. Except for basic product quality, the comprehensive experience, service, and other characteristics are also principal elements affecting consumers' perception of product quality. The main service measures in the physical showroom consist of advertising, product deployment, and introducing advanced technology, such as VR glasses. Through the physical showroom, consumers have an insightful understanding of products in diversified consumption scenarios, thus increase perceptions of product quality.

Besides, product quality not only plays a key role in consumer purchase decision, but also has a significant impact on the service decision in the physical showroom [21]. For example, electronics makers, such as Apple, and Huawei, often focus on high-quality products in the physical showroom. However, appliance makers may pay more attention to medium-quality products according to the GFK's report ${ }^{3}$. The cases show that product quality is a major driver before making the showroom service decision. Thus, we investigate the service decision and pricing based on product quality in the three showroom strategies. With all the above observations in mind, we seek to understand the following: (1) How does product quality affect the physical showroom operation? (2) What is the optimal service decision and pricing in three showroom strategies? (3) What is the optimal operation strategy of the physical showroom?

To solve the above question, an analytical model is proposed to capture the basic characteristics of consumer purchase behaviors under the physical showroom. Then, we obtain and compare the equilibrium solutions of the optimal services and prices based on product quality in the $\mathrm{CO}, \mathrm{MO}$, and $\mathrm{RO}$ strategies. Besides, we relax the exogenous assumption of product quality and analyze the solution when product quality is endogenous. Finally, the robustness of the models is verified by discussing high consumer heterogeneity and a partial refund policy.

The contributions of this paper are threefold. First, conventional wisdom suggests that the high-quality product should be offered a high service than the low-quality product. Interestingly, we find that the optimal showroom service may descend with the increase of product quality when both the product quality and the production cost coefficient are high. Second, the optimal operation strategy is acquired based on product quality. When the production cost coefficient is low, both the MO strategy and the RO strategy are better than the CO

\footnotetext{
$1_{\text {https: }} / /$ www .made.com/showrooms.

$2_{\text {http: } / / \text { ww . cww.net.cn/article?id=460759. }}$

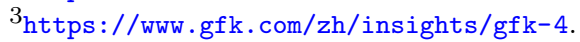


strategy. The enterprise (the manufacturer or the e-retailer) should open the physical showroom separately than jointly. When the production cost coefficient is high, the CO strategy provides the optimal showroom service. The enterprises in the supply chain should cooperate to balance costs and benefits. Third, when product quality is endogenous, the RO strategy may provide better product quality than the CO strategy if the production cost coefficient is moderate. In other words, the decentralized channel may produce higher product quality than the centralized channel when the e-retailer provides showroom service.

The remainder of this paper is organized as follows. Section 2 reviews the relevant literature. Section 3 presents the problem description. Section 4 builds the models and analyzes the equilibrium in the three strategies. The endogenous quality and model extension are discussed in Sections 5 and 6. Finally, Section 7 concludes this paper.

\section{LiterATURE REVIEW}

This paper is primarily related to three streams of literature: (a) the showroom strategy in omnichannel operation, (b) the channel operation, and (c) the product quality decision in the supply chain.

\subsection{Showroom strategy}

The showroom strategy, as one of the most popular omnichannel strategies, has received a great deal of attention over the past serval years. The physical showroom breaks the boundary between brick-and-mortar and online channels [35], thus being popular in e-business. Enterprises operate the physical showroom in an integrated manner, aiming at providing an undifferentiated purchasing experience for consumers while avoiding channel conflict $[6,32]$.

This paper focuses on the showroom strategy in omnichannel operation, which stands for a retailing service to keep up with consumer experience needs [19]. To examine the effectiveness of the showroom strategy, some researchers study the showroom strategy from an empirical perspective. Gensler et al. [21] survey 556 respondents to examine how showrooms influence consumer decisions. The results find that non-price factors also play a pivotal role in consumer decisions: perceived product quality and waiting time in the brick-and-mortar store. Fernndez et al. [16] evaluate the influence of showrooms based on a specific case of products purchased online, and the results illustrate that the showroom may improve the quality value of products. Fassnacht et al. [15] address the effect of the showroom service based on three scenario experiments, and the results indicate that high showroom service increases purchase intentions.

Others build analytical models to study the showroom strategy. Bell et al. [3,4] point out that the physical showroom increases online demand and generates operational spillovers to the offline channel. Thus, overall operational efficiency is improved. Gao and $\mathrm{Su}[19]$ consider three information mechanisms: physical showrooms, virtual showrooms, and availability information in omnichannel operation. The results indicate that physical showrooms may induce retailers to reduce store inventory. Gallino et al. [18] prove that the application of virtual technologies in physical showrooms decreases return rates. Du et al. [13] suggest that the physical showroom may offset the negative impact of stock-out disappointment and improve the retailer's profit. And Fan et al. [14] develop a game-theoretical model to help the retailer choose a pure showroom or a selling store. The authors suggest that when the proportion of the store consumer segment is not too high, one e-retailer may launch the pure showroom, and the other may open the selling store. Besides, Zhang et al. [41] study the assortment service of online products in the physical showroom. Li et al. [27] find that the partial assortment service may be more profitable than the full assortment service. In summary, extant works on the showroom strategy focus on the efficiency of the physical showroom, such as reducing online return rate, expanding market demand, and increasing total efficiency. However, there is a lack of literature to study the optimal operation strategy of showrooms. 


\subsection{Channel operation}

The second relevant literature stream investigates how channel operation affects the performance of the supply chain. In particular, our study is highly related to the three structures: the MO, the RO, and the CO [8]. Chiang et al. [12] study the pricing decision for the manufacturer with online and offline channels. The results show that the MO strategy is better for the manufacturer. Hsiao and Chen [25] compare the three structures and suggest that each kind of channel structure may become the dominant strategy according to the number of loyal customers. Abhishek et al. [1] explore the direct selling strategy and agency selling strategy in electronic and brick-and-mortar. The results show that online spillover has a strong impact on the decisions to open channels. Under different power structures, Chen et al. [9] obtain and compare the optimal operational decisions when the retailer operates offline and online channels. Xue and Zhang [38] study a supply chain with manufacturer encroachment where product quality is an endogenous decision. Besides, Yang and Zhang [39] explore the operation strategies for an omnichannel supply chain by establishing and comparing four scenarios. The results show that the omnichannel strategy generates benefits for the firms in many situations.

To sum up, previous works have placed a great emphasis on the traditional retailing scenario. Unlike the prior research, we study the operation strategies under omnichannel retailing, which emphasizes service cooperation in the offline and online channels. Particularly, we focus on the optimal operation strategy of the physical showroom in this paper.

\subsection{Product quality decision}

The third related stream of literature is the product quality decision in the supply chain. In contrast to the inadequate showroom service literature, the literature on the product quality decision is comparatively mature. Villas-Boas [36] and Jeuland and Shugan [26] validate that product quality in the decentralized channel is lower than that in the centralized channel because of the double marginal effect. Shi et al. [33] find that when consumer heterogeneity follows the general distribution of consumers' willingness to pay (WTP), a decentralized channel may lead to a higher product quality level. Ha et al. [24] suggest that a manufacturer may prefer to sell its high-quality product in the direct channel rather than in the decentralized distribution channel when product quality is endogenous.

Moreover, some papers focus on the joint analysis of service, pricing, and product quality in the supply chain. $\mathrm{Xu}$ [37] and Chen et al. [10] propose a joint pricing and product quality decision in a distribution channel. Giri et al. [22] study the product quality and pricing issue in a closed-loop supply chain. Chen et al. [11] focus on retail service, manufacturer's direct service and quality effort, and present an analytical framework to examine the optimal decisions in a dual-channel supply chain. Fu et al. [17] investigate the quality and pricing decisions in a supply chain consisting of one retailer and two manufacturers. In summary, a lot of papers study joint research on service, pricing, and product quality under traditional retailing, but few research results focus on the joint decision under omnichannel retailing.

Previous studies have extensively researched the pricing decision considering retail service or manufacturer service in a traditional supply chain. Different from the above-reviewed literature, this paper analyzes showroom operation strategy and pricing in the omnichannel supply chain. Meanwhile, motivated by the rapid development of the physical showroom, joint decisions of showroom service and pricing based on product quality are analyzed under three typical channel structures. To our knowledge, there is no literature studying the showroom service decision based on product quality. In addition, by relaxing the assumption that product quality is exogenous, we compare the optimal product quality among the three strategies when product quality is endogenous.

\section{Problem Description}

We consider a supply chain consisting of a manufacturer (he), an e-retailer (she), and consumers. Then we describe them to capture their essential features. 
(a)

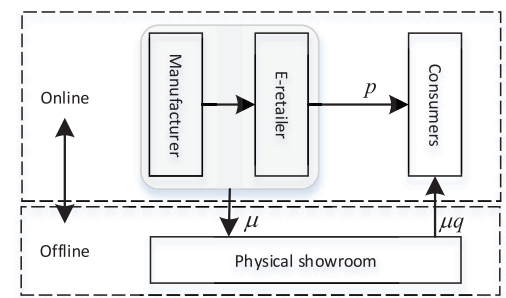

(b)

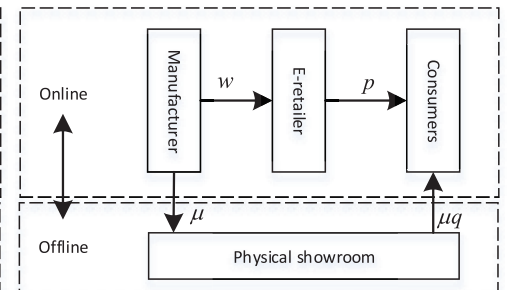

(c)

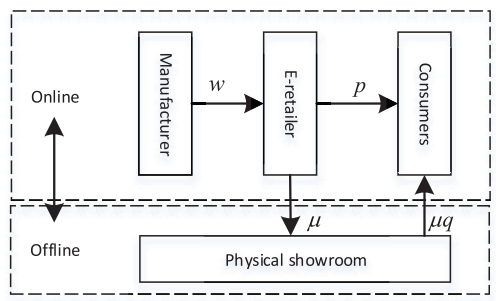

FiguRE 1. Three operation strategies of the physical showroom. (a) The CO strategy. (b) The MO strategy. (c) The RO strategy.

Firms. He produces a product with exogenous quality $q$. The production cost is $k q^{2}$, where $k$ represents the production cost coefficient. Let $w$ and $p$ stand for the wholesale price and the retail price respectively. $\mu \sim U[0,1]$ denotes the showroom service and the cost is $\frac{1}{2} h \mu^{2}$, where $h$ is the showroom cost coefficient [31]. Also, we adopt $s q$ to represent the salvage value of online return product $[28,34]$, where $s$ denotes the salvage value coefficient. Besides, a full refund policy is allowed by the upstream and downstream.

Consumers. Consumers are assumed to have a unit demand in the market. Without loss of generality, we normalize the number of consumers to one. Let $\theta$ denote the consumer's WTP for product quality $q$. And $\theta$ is distributed uniformly over $[x, 1]$, which $x$ is large enough to ensure that the product can cover the whole market. As consumers are heterogeneous to product quality, a proportion $\alpha \in(0,1)$ of consumers has positive value $\theta q$ and a fraction of $1-\alpha$ has zero value. The former is named "high-type" and the latter is described as "low-type". High-type consumers buy products directly without return and low-type consumers choose to return products after purchasing. Consumer expected return cost is assumed to be 0 . Let $\mu q$ represent positive influence of the showroom service on consumer's utility. Therefore, the consumer's expected payoff is described as: $U(\theta)=\alpha(\theta q-p)+(1-\alpha) \cdot 0+\mu q$. Set $U(\theta)=0$, the consumer's WTP is $\theta^{*}=\frac{\alpha p-\mu q}{\alpha q}$, and $\theta \in\left[\max \left\{\theta^{*}, x\right\}, 1\right]$. Consequently, the demand function is calculated as $D=\min \left\{\frac{1}{1-x}\left(1-\frac{\alpha p-\mu q}{\alpha q}\right), 1\right\}$.

Strategies. Three operation strategies of the physical showroom are considered under e-commerce: the CO strategy, the MO strategy, and the RO strategy, as shown in Figure 1. The CO strategy means that the manufacturer and the e-retailer operate the physical showroom jointly. The MO strategy represents that the manufacturer opens the physical showroom. And the RO strategy denotes that the e-retailer operates the physical showroom.

Sequence of events. (1) In the CO strategy, they collectively decide the retail price $p$ and service decision $\mu$. Then consumers make their purchase decisions. (2) In the MO strategy, he initially decides the wholesale price $w$ and service decision $\mu$. Then she sells the product through the online channel at the retail price $p$. Consumers make their purchase decisions. (3) In the RO strategy, given the wholesale price $w$, she decides the service decision $\mu$ and the retail price $p$. Consumers make their purchase decisions.

\section{Strategy analysis}

\subsection{Cooperative operation $(\mathrm{CO})$}

The CO strategy means that the manufacturer and the e-retailer operate the physical showroom and make decisions jointly. The total profit is described as $\pi_{s c}^{c}=\left[\alpha\left(p-k q^{2}\right)+(1-\alpha)\left(s q-k q^{2}\right)\right] D-\frac{1}{2} h \mu^{2}$. Given the demand function $D=\min \left\{\frac{1}{1-x}\left(1-\frac{\alpha p-\mu q}{\alpha q}\right), 1\right\}$, backward induction is embraced to solve the model. By maximizing the profit, the optimal price $p^{c^{*}}$ is found firstly, and then the optimal service decision $\mu^{c^{*}}$ is obtained. The results are shown in Lemma 4.1. 
Lemma 4.1. In the $M O$ strategy,

(1) when $k \leq \frac{(1-\alpha) s+\alpha}{2(1-\alpha) \alpha h}$, if $q \leq \frac{[(2 x-1) \alpha+(1-\alpha) s] h}{h k-1}, \mu^{c^{*}}=\frac{q}{h}$, and $p^{c^{*}}=\frac{q^{2}+x a h q}{\alpha h}$; if $\frac{[(2 x-1) \alpha+(1-\alpha) s] h}{h k-1}<q \leq$ $\frac{(2 x-1) \alpha+(1-\alpha) s+1}{k}, \mu^{c^{*}}=k q-(2 x-1) \alpha-(1-\alpha) s$, and $p^{c^{*}}=\frac{k q^{2}+(1-x) \alpha q-(1-\alpha) s q}{\alpha}$; otherwise, $\mu^{c^{*}}=1$, and $p^{c^{*}}=\frac{\alpha x q+q}{\alpha}$

(2) when $k>\frac{(1-\alpha) s+\alpha}{2(1-\alpha) \alpha h}$, if $q \leq \frac{[(2 x-1) \alpha+(1-\alpha) s] h}{h k-1}, \mu^{c^{*}}=\frac{q}{h}$, and $p^{c^{*}}=\frac{q^{2}+x a h q}{\alpha h}$; if $\frac{[(2 x-1) \alpha+(1-\alpha) s] h}{h k-1}<q \leq$ $\frac{\alpha+(1-\alpha) s}{k}, \mu^{c^{*}}=\frac{\alpha q+(1-\alpha) s q-k q^{2}}{2(1-x) \alpha h-q}$, and $p^{c^{*}}=\frac{[(1-x) \alpha h k+(1-\alpha) s] q^{2}+(1-x) \alpha h[\alpha-(1-\alpha) s] q-k q^{3}}{\alpha[2(1-x) \alpha h-q]}$; otherwise, no product is produced.

Proof. In the CO strategy, the product demand is $D=\min \left\{\frac{1}{1-x}\left(1-\frac{\alpha p-\mu q}{\alpha q}\right), 1\right\}$. By solving $\frac{\partial^{2} \pi_{s c}^{c}}{\partial p^{2}}$, we firstly obtain the solution $\frac{\partial^{2} \pi_{s c}^{c}}{\partial p^{2}}=-\frac{2 \alpha}{(1-x) q}<0$, which indicates that $\pi_{s c}^{c}$ is a strictly concave function of $p$. Then, by solving $\frac{\partial \pi_{s c}^{c}}{\partial p}=0$, the optimal retail price is $p^{c^{*}}=\frac{k q^{2}+(\alpha+\mu) q-(1-\alpha) s q}{2 \alpha}$, and the consumer's WTP is $\theta^{*}=$ $\frac{k q+(\alpha+\mu)-(1-\alpha) s}{2 \alpha}$. Therefore, we find the best responses of the e-retailer: (1) When $\mu<k q-(2 x-1) \alpha-(1-\alpha) s$, $p^{c^{*}}=\frac{k q^{2}+(\alpha+\mu) q-(1-\alpha) s q}{2 \alpha}, D^{c^{*}}=\frac{\alpha+\mu+(1-\alpha) s-k q}{2(1-x) \alpha}$, and $\pi_{s c}^{c^{*}}=\frac{q[\alpha+\mu+(1-\alpha) s-k q]^{2}}{4(1-x) \alpha}-\frac{1}{2} h \mu^{2}$. (2) When $\mu \geq k q-$ $(2 x-1) \alpha-(1-\alpha) s, p^{c^{*}}=\frac{\alpha x q+\mu q}{\alpha}, D^{c^{*}}=1$, and $\pi_{s c}^{c^{*}}=(x \alpha+\mu) q+(1-\alpha) s q-k q^{2}-\frac{1}{2} h \mu^{2}$.

Then, by solving $\frac{\partial^{2} \pi_{s c}^{c^{*}}}{\partial \mu^{2}}$ in the above two situations, we find that when $\mu<k q-(2 x-1) \alpha-(1-\alpha) s$, $\frac{\partial^{2} \pi_{s c}^{c^{*}}}{\partial \mu^{2}}=\frac{q-2(1-x) \alpha h}{2(1-x) \alpha}<0$ if $q<2(1-x) \alpha h$. Besides, we discuss the ranges of $q$ in the following, and make sure that the constraint $q<2(1-x) \alpha h$ is always satisfied; When $\mu \geq k q-(2 x-1) \alpha-(1-\alpha) s, \frac{\partial^{2} \pi_{s c}^{c^{*}}}{\partial \mu^{2}}=-h<0$. The above results indicate that $\pi_{s c}^{c^{*}}$ is a strictly concave function of $\mu$. By solving $\frac{\partial \pi_{s c}^{c^{*}}}{\partial \mu}=0$, the results are divided into the following two parts.

(1) When $\mu<k q-(2 x-1) \alpha-(1-\alpha) s$, the optimal showroom service is $\mu^{c^{*}}=\frac{\alpha q+(1-\alpha) s q-k q^{2}}{2(1-x) \alpha h-q}$ if $q \leq \frac{\alpha+(1-\alpha) s}{k}$. Given $\frac{\alpha+(1-\alpha) s}{k}<2(1-x) \alpha h$, we obtain $k>\frac{(1-\alpha) s+\alpha}{2(1-\alpha) \alpha h}$. Considering the constraint $0<\mu^{c^{*}} \leq \mu$, we derive that $q>\frac{[(2 x-1) \alpha+(1-\alpha) s] h}{h k-1}$. Therefore, if $q \leq \frac{[(2 x-1) \alpha+(1-\alpha) s] h}{h k-1}, \mu^{c^{*}}=k q-(2 x-1) \alpha-(1-\alpha) s$; If $\frac{[(2 x-1) \alpha+(1-\alpha) s] h}{h k-1}<q \leq \frac{\alpha+(1-\alpha) s}{k}, \mu^{c^{*}}=\frac{\alpha q+(1-\alpha) s q-k q^{2}}{2(1-x) \alpha h-q}$; Otherwise, the manufacturer does not sell the product. Besides, let $h \geq \frac{1}{k}$ ensure that $q \leq \frac{[(2 x-1) \alpha+(1-\alpha) s] h}{h k-1}$ makes sense.

(2) When $\mu \geq k q-(2 x-1) \alpha-(1-\alpha) s$, the optimal showroom service is $\mu^{c^{*}}=\frac{q}{h}$. Considering $\mu \leq \mu^{c^{*}}$, we find that if $q \leq \frac{[(2 x-1) \alpha+(1-\alpha) s] h}{h k-1}, \mu^{c^{*}}=\frac{q}{h}$; if $\frac{[(2 x-1) \alpha+(1-\alpha) s] h}{h k-1}<q \leq \frac{(2 x-1) \alpha+(1-\alpha) s+1}{k}, \mu^{c^{*}}=k q-$ $(2 x-1) \alpha-(1-\alpha) s$; otherwise, $\mu^{c^{*}}=1$.

In conclusion, Lemma 4.1 is obtained. Proof ends.

Lemma 4.1 states the equilibrium of the optimal service and price based on product quality in the CO strategy. According to Lemma 4.1, the trends of the optimal showroom service are different with diverse production cost coefficients. when the production cost coefficient is low, they tend to continuously enhance service quality with product quality. However, when the production cost coefficient is high, they prefer to adopt a low showroom service to balance the service cost and incomes if product quality is relatively high. Hence, the service decision may decrease with the increase of product quality. Besides, when product quality is beyond a threshold $\left(q>\frac{\alpha+(1-\alpha) s}{k}\right)$, the manufacturer does not provide the product. According to the equilibrium in Lemma 4.1, an interesting result is obtained in Proposition 4.2.

Proposition 4.2. In the CO strategy, the optimal showroom service may decrease with the increase of product quality when the production cost coefficient is high. Formally, when $k>\frac{(1-\alpha) s+\alpha}{2(1-\alpha) \alpha h}$, if 
(a)

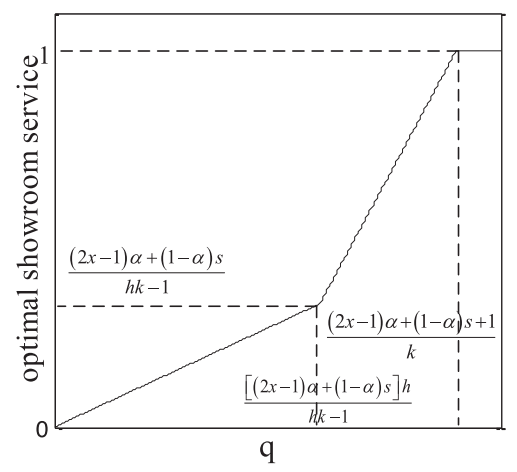

(b)

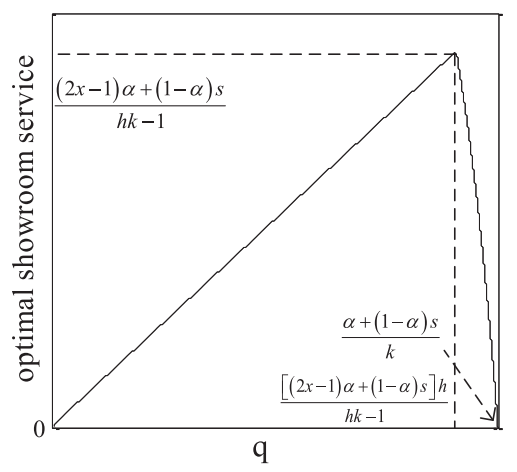

Figure 2. The optimal showroom service in the CO strategy. (a) When $k$ is low. (b) When $k$ is high.

$\max \left\{\frac{[(2 x-1) \alpha+(1-\alpha) s] h}{h k-1}, \widehat{q}\right\}<q \leq \frac{\alpha+(1-\alpha) s}{k}, \frac{\partial \mu^{c^{*}}}{\partial q}<0$, where $\widehat{q}$ is the smaller solution of $k q^{2}+$ $2(1-x) \alpha h[\alpha+(1-\alpha) s-2 k q]=0$.

Proof. According to Lemma 4.1, when $k>\frac{(1-\alpha) s+\alpha}{2(1-\alpha) \alpha h}$, if $\frac{[(2 x-1) \alpha+(1-\alpha) s] h}{h k-1}<q \leq \frac{\alpha+(1-\alpha) s}{k}$, let $\frac{\partial \mu^{c^{*}}}{\partial q}=$ $\frac{k q^{2}+2(1-x) \alpha h[\alpha+(1-\alpha) s-2 k q]}{[2(1-x) \alpha h-q]^{2}}=0$, and we obtain the equation $k q^{2}+2(1-x) \alpha h[\alpha+(1-\alpha) s-2 k q]=0$. We find that $\Delta>0$, and denote the equation has two roots: $\widehat{q}$ and $\breve{q}(0<\widehat{q}<\breve{q})$. When $q \in(\widehat{q}, \breve{q}), \frac{\partial \mu^{c^{*}}}{\partial q}<0$. Therefore, when $\max \left\{\frac{[(2 x-1) \alpha+(1-\alpha) s] h}{h k-1}, \widehat{q}\right\}<q \leq \frac{\alpha+(1-\alpha) s}{k}, \mu^{c^{*}}=\frac{\alpha q+(1-\alpha) s q-k q^{2}}{2(1-x) \alpha h-q}$ is a monotonically decreasing function, where $\widehat{q}$ is the smaller solution of $k q^{2}+2(1-x) \alpha h[\alpha+(1-\alpha) s-2 k q]=0$. Proof ends.

Conventional wisdom believes that the high-quality product should be offered a high service than the lowquality product. Interestingly, we find that the optimal showroom service decision shows a descending trend when both product quality and the production cost coefficient are high. The reason is that consumers are more sensitive to the retail price than the showroom service when buying high-quality products, such as furniture and household appliances. Meanwhile, as the production cost coefficient is also high, enterprises tend to choose a lower service level to balance the costs and benefits, thus maximizing their marginal profits. Consequently, the optimal showroom service may decrease with the increase of product quality.

Figure 2 illustrates the service decision equilibrium in Lemma 4.1. In the low $k$ case, the showroom service decision is positively related to product quality. Especially, the growth rate of showroom service increases significantly along with product quality. However, in the high $k$ case, the showroom service increases firstly and then decreases. It is concluded that the showroom service decision is related to different production cost coefficient. To sum up, enterprises should consider both product quality and production cost before making the showroom service decision in the CO strategy. In particular, when the production cost coefficient is low, enterprises should pay more attention to high-quality products. But when the production cost coefficient is high, enterprises should focus on medium-quality products.

\subsection{Manufacturer operation (MO)}

The MO strategy represents that the manufacturer operates the physical showroom. In the MO strategy, the manufacturer's profit is $\pi_{m}^{m}=\left[\alpha\left(w-k q^{2}\right)+(1-\alpha)\left(s q-k q^{2}\right)\right] D-\frac{1}{2} h \mu^{2}$, and the e-retailer's profit is $\pi_{r}^{m}=\alpha(p-w) D$. Backward induction is adopted to solve the model. First, we obtain the e-retailer's best responses: the optimal retail price $p^{m^{*}}$, the product demand $D^{m^{*}}$ and the e-retailer's optimal profit $\pi_{r}^{m^{*}}$. Then, 
the optimal wholesale price $w^{m^{*}}$ is obtained based on the principle of the manufacturer's profit maximization. In conclusion, the results are shown in Lemma 4.3.

Lemma 4.3. In the $M O$ strategy,

(1) when $k \leq \frac{(1-\alpha) s+\alpha}{4(1-\alpha) \alpha h}$, if $q \leq \frac{[(4 x-3) \alpha+(1-\alpha) s] h}{h k-1}, \mu^{m^{*}}=\frac{q}{h}, w^{m^{*}}=\frac{q^{2}+(2 x-1) \alpha h q}{\alpha h}$, and $p^{m^{*}}=\frac{q^{2}+x \alpha h q}{\alpha h}$; if $\frac{[(4 x-3) \alpha+(1-\alpha) s] h}{h k-1}<q \leq \frac{(4 x-3) \alpha+(1-\alpha) s+1}{k}, \mu^{m^{*}}=k q-(4 x-3) \alpha-(1-\alpha) s, w^{m^{*}}=\frac{k q^{2}+2(1-x) \alpha q-(1-\alpha) s q}{\alpha}$, and $p^{m^{*}}=\frac{k q^{2}+3(1-x) \alpha q-(1-\alpha) s q}{\alpha}$; otherwise, $\mu^{m^{*}}=1, w^{m^{*}}=\frac{(2 x-1) \alpha q+q}{\alpha}$, and $p^{m^{*}}=\frac{x \alpha q+q}{\alpha} ;$

(2) when $k>\frac{(1-\alpha) s+\alpha}{2(1-\alpha) \alpha h}$, if $q \leq \frac{[(4 x-3) \alpha+(1-\alpha) s] h}{h k-1}, \quad \mu^{m^{*}}=\frac{q}{h}, \quad w^{m^{*}}=\frac{q^{2}+(2 x-1) \alpha h q}{\alpha h}$, and $p^{m^{*}}=\frac{q^{2}+x \alpha h q}{\alpha h} ; \quad$ if $\frac{[(4 x-3) \alpha+(1-\alpha) s] h}{h k-1}<q \leq \frac{\alpha+(1-\alpha) s}{k}, \quad \mu^{m^{*}}=\frac{\alpha q+(1-\alpha) s q-k q^{2}}{4(1-x) \alpha h-q}$, $w^{m^{*}}=\frac{[2(1-x) \alpha h k+(1-\alpha) s] q^{2}+2(1-x) \alpha h[\alpha-(1-\alpha) s] q-k q^{3}}{\alpha[4(1-x) \alpha h-q]}$, and $p^{m^{*}}=\frac{[(1-x) \alpha h k+(1-\alpha) s] q^{2}+(1-x) \alpha h[3 \alpha-(1-\alpha) s] q-k q^{3}}{\alpha[4(1-x) \alpha h-q]}$; otherwise, no product is produced.

Proof. In the MO strategy, the e-retailer's profit is $\pi_{r}^{m}=\alpha(p-w) D$. By solving $\frac{\partial^{2} \pi_{r}^{m}}{\partial p^{2}}$, we find that $\frac{\partial^{2} \pi_{r}^{m}}{\partial p^{2}}=$ $-\frac{2 \alpha}{(1-x) q}<0$, which reveals that $\pi_{r}^{m}$ strictly concave function of $p$. Then, let $\frac{\partial \pi_{r}^{m}}{\partial p}=0$ and we obtain $p^{m^{*}}=$ $\frac{(\alpha+\mu) q+\alpha w}{2 \alpha}$. Hence, the best responses of the e-retailer are as follows: (1) When $\mu<\frac{\alpha w-(2 x-1) \alpha q}{q}, p^{m^{*}}=$ $\frac{(\alpha+\mu) q+\alpha w}{2 \alpha}, D^{m^{*}}=\frac{(\alpha+\mu) q-\alpha w}{2(1-x) \alpha q}$ and $\pi_{r}^{m^{*}}=\frac{[(\alpha+\mu) q-\alpha w]^{2}}{4(1-x) \alpha q} ;(2)$ When $\mu \geq \frac{\alpha w-(2 x-1) \alpha q}{q}, p^{m^{*}}=\frac{\alpha x q+\mu q}{\alpha}, D^{m^{*}}=1$, and $\pi_{r}^{m^{*}}=(x \alpha+\mu) q-\alpha w$.

Then, we plug the demand function $D^{m^{*}}$ into the function $\pi_{m}^{m}=\left[\alpha\left(w-k q^{2}\right)+(1-\alpha)\left(s q-k q^{2}\right)\right] D-\frac{1}{2} h \mu^{2}$. By solving $\frac{\partial^{2} \pi_{m}^{m}}{\partial w^{2}}$, we obtain that $\frac{\partial^{2} \pi_{m}^{m}}{\partial w^{2}}=-\frac{\alpha}{(1-x) q}<0$ when $\mu<\frac{\alpha w-(2 x-1) \alpha q}{q}$, which reveals that $\pi_{m}^{m}$ is a strictly concave function of $w$, and $\pi_{m}^{m}$ is an increasing function when $\mu \geq \frac{\alpha w-(2 x-1) \alpha q}{q}$. The optimal wholesale price $w^{m^{*}}$ is described in the following. (1) When $\mu<\frac{\alpha w-(2 x-1) \alpha q}{q}, w^{m^{*}}=\frac{k q^{2}+(\alpha+\mu) q-(1-\alpha) s q}{2 \alpha}, D^{m^{*}}=\frac{\alpha+\mu+(1-\alpha) s-k q}{4(1-x) \alpha}$, and $\pi_{m}^{m^{*}}=\frac{\left[(\alpha+\mu) q+(1-\alpha) s q-k q^{2}\right]^{2}}{8(1-x) \alpha q}-\frac{1}{2} h \mu^{2} ;(2)$ When $\mu \geq \frac{\alpha w-(2 x-1) \alpha q}{q}, w^{m^{*}}=\frac{(2 x-1) \alpha q+\mu q}{\alpha} D^{m^{*}}=1$, and $\pi_{m}^{m^{*}}=$ $(2 x-1) \alpha q+\mu q+(1-\alpha) s q-k q^{2}-\frac{1}{2} h \mu^{2}$.

Third, by solving $\frac{\partial^{2} \pi_{m}^{m^{*}}}{\partial \mu^{2}}$, when $\mu<k q-(4 x-3) \alpha-(1-\alpha) s$, the solution $\frac{\partial^{2} \pi_{m}^{m^{*}}}{\partial \mu^{2}}=\frac{q-4(1-x) \alpha h}{4(1-x) \alpha}<0$ if $q<4(1-x) \alpha h$. We discuss the ranges of $q$ in the following, and make sure that the constraint $q<4(1-x) \alpha h$ is satisfied; When $\mu \geq k q-(4 x-3) \alpha-(1-\alpha) s$, the solution $\frac{\partial^{2} \pi_{s c}^{c^{*}}}{\partial \mu^{2}}=-h<0$. The above results indicate that $\pi_{s c}^{c^{*}}$ is a strictly concave function of $\mu$. Then, we derive the optimal showroom service $\mu^{m^{*}}$.

(1) When $\mu<k q-(4 x-3) \alpha-(1-\alpha) s, \mu^{m^{*}}=\frac{\alpha q+(1-\alpha) s q-k q^{2}}{4(1-x) \alpha h-q}$ if $q<4(1-x) \alpha h$ and $k>\frac{(1-\alpha) s+\alpha}{4(1-\alpha) \alpha h}$. Considering $0<\mu^{m^{*}} \leq \mu$, we obtain that $q>\frac{[(4 x-3) \alpha+(1-\alpha) s] h}{h k-1}$. As a result, if $q \leq \frac{[(4 x-3) \alpha+(1-\alpha) s] h}{h k-1}$, $\mu^{m^{*}}=k q-(4 x-3) \alpha-(1-\alpha) s$; if $\frac{[(4 x-3) \alpha+(1-\alpha) s] h}{h k-1}<q \leq \frac{\alpha+(1-\alpha) s}{k}, \mu^{m^{*}}=\frac{\alpha q+(1-\alpha) s q-k q^{2}}{4(1-x) \alpha h-q}$. Otherwise, the manufacturer does not produce the product.

(2) When $\mu \geq k q-(4 x-3) \alpha-(1-\alpha) s, \mu^{m^{*}}=\frac{q}{h}$. Similarly, if $q \leq \frac{[(4 x-3) \alpha+(1-\alpha) s] h}{h k-1}, \mu^{m^{*}}=\frac{q}{h}$; if $\frac{[(4 x-3) \alpha+(1-\alpha) s] h}{h k-1}<q \leq \frac{(4 x-3) \alpha+(1-\alpha) s+1}{k}, \mu^{m^{*}}=k q-(4 x-3) \alpha-(1-\alpha) s$. Otherwise, $\mu^{m^{*}}=1$.

To sum up, the optimal solutions are obtained in Lemma 4.3. Proof ends.

Lemma 4.3 illustrates the equilibrium of the showroom service decision, the wholesale price, and the retail price based on product quality in the MO strategy. Similar to the CO strategy, the trends of the showroom service are also different with low and high production cost coefficients. According to Lemma 4.3, we obtain Proposition 4.4 in the following.

Proposition 4.4. In the MO strategy, the optimal showroom service may decrease with the increase of product quality when the production cost coefficient is high. Formally, when $k>\frac{(1-\alpha) s+\alpha}{4(1-\alpha) \alpha h}$, if 
(a)

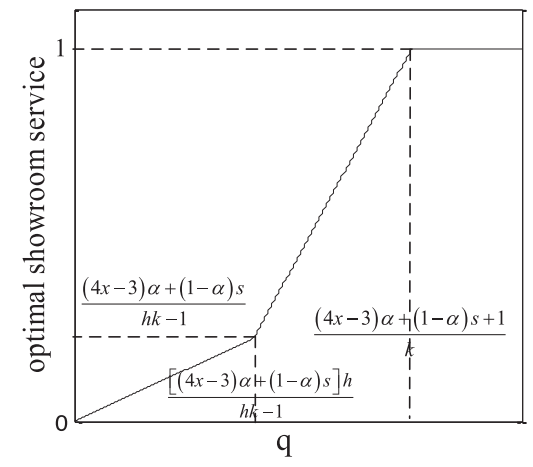

(b)

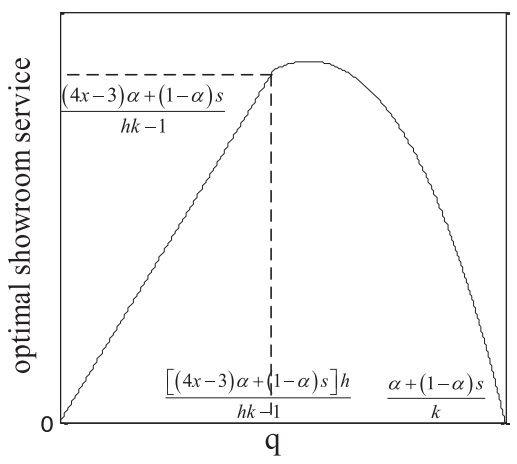

Figure 3. The optimal showroom service in the MO strategy. (a) When $k$ is low. (b) When $k$ is high.

$\max \left\{\frac{[(4 x-3) \alpha+(1-\alpha) s] h}{h k-1}, \widehat{q}^{m}\right\}<q \leq \frac{\alpha+(1-\alpha) s}{k}, \frac{\partial \mu^{m^{*}}}{\partial q}<0$, where $\widehat{q}^{m}$ is the smaller solution of $k q^{2}+$ $4(1-x) \alpha h[\alpha+(1-\alpha) s-2 k q]=0$.

Proof. According to Lemma 4.3, when $k>\frac{(1-\alpha) s+\alpha}{4(1-\alpha) \alpha h}$, if $\frac{[(4 x-3) \alpha+(1-\alpha) s] h}{h k-1}<q \leq \frac{\alpha+(1-\alpha) s}{k}$, let $\frac{\partial \mu^{m^{*}}}{\partial q}=$ $\frac{k q^{2}+4(1-x) \alpha h[\alpha+(1-\alpha) s-2 k q]}{[4(1-x) \alpha h-q]^{2}}=0$ and we obtain the equation $k q^{2}+4(1-x) \alpha h[\alpha+(1-\alpha) s-2 k q]=0$. We find that $\Delta>0$, and denote the equation has two roots: $\widehat{q}^{m}$ and $\breve{q}^{m}\left(0<\widehat{q}^{m}<\breve{q}^{m}\right)$. When $q \in\left(\widehat{q}^{m}, \breve{q}^{m}\right)$, $\frac{\partial \mu^{c^{*}}}{\partial q}<0$. Therefore, when $\max \left\{\frac{[(4 x-3) \alpha+(1-\alpha) s] h}{h k-1}, \stackrel{m}{q}\right\}<q \leq \frac{\alpha+(1-\alpha) s}{k}, \mu^{m^{*}}=\frac{\alpha q+(1-\alpha) s q-k q^{2}}{4(1-x) \alpha h-q}$ is a monotonically decreasing function, where $\widehat{q}^{m}$ is the smaller solution of $k q^{2}+4(1-x) \alpha h[\alpha+(1-\alpha) s-2 k q]=0$. Proof ends.

Comparing with Lemmas 4.1 and 4.3, we find that the trends of the showroom service in the MO strategy are similar to that in the CO strategy. As shown in Figure 3, when the production cost coefficient is low, the optimal showroom service increases along with product quality. When the production cost coefficient is high, the optimal showroom service increases firstly and then decreases with product quality.

The differences between the MO strategy and the CO strategy are as follows. When the production cost coefficient is high, the manufacturer undertakes the total costs of product production and showroom operation in the MO strategy, leading to free-riding of the e-retailer. Owing to the double marginal effect, the optimal showroom service provided in the MO strategy is usually lower than that in the CO strategy. However, when the production cost coefficient is low, contrary to common sense, the MO strategy may provide better showroom service than the CO strategy, as illustrated in Corollary 4.5.

Corollary 4.5. When the production cost coefficient is low, the MO strategy may offer a higher service than the CO strategy. Formally, when $k \leq \frac{(1-\alpha) s+\alpha}{4(1-\alpha) \alpha h}, \mu^{m^{*}} \geq \mu^{c^{*}}$.

Proof. When $k \leq \frac{(1-\alpha) s+\alpha}{4(1-\alpha) \alpha h}$, if $q \leq \frac{[(4 x-3) \alpha+(1-\alpha) s] h}{h k-1}, \mu^{m^{*}}=\mu^{c^{*}} ;$ if $\frac{[(4 x-3) \alpha+(1-\alpha) s] h}{h k-1}<q \leq \frac{[(2 x-1) \alpha+(1-\alpha) s] h}{h k-1}$, $\mu^{m^{*}}-\mu^{c^{*}}=k q-(4 x-3) \alpha-(1-\alpha) s-\frac{q}{h}>0$; if $\frac{[(2 x-1) \alpha+(1-\alpha) s] h}{h k-1}<q \leq \frac{(4 x-3) \alpha+(1-\alpha) s+1}{k}, \mu^{m^{*}}-\mu^{c^{*}}=k q-$ $(4 x-3) \alpha-(1-\alpha) s-[k q-(2 x-1) \alpha-(1-\alpha) s]=(1-x) \alpha>0$; if $\frac{(4 x-3) \alpha+(1-\alpha) s+1}{k}<q \leq \frac{(2 x-1) \alpha+(1-\alpha) s+1}{k}$, $\mu^{m^{*}}-\mu^{c^{*}}=1-[k q-(2 x-1) \alpha-(1-\alpha) s]>0$; otherwise, $\mu^{m^{*}}=\mu^{c^{*}}$. Therefore, $\mu^{m^{*}} \geq \mu^{c^{*}}$. Proof ends.

Corollary 4.5 diverges from conventional intuition that the CO strategy offers a better service. We find that the MO strategy may offer a better service than the CO strategy when the production cost coefficient is low. 
The reason is that, when the manufacturer produces higher-quality products with the low production cost coefficient, he has more incentive to improve showroom service to expand market share and maximize profits. Consequently, when the production cost coefficient is low, the manufacturer should open the physical showroom separately than jointly. When the production cost coefficient is high, the situation is quite the opposite. The manufacturer should cooperate with the e-retailer to balance costs and benefits.

\subsection{E-retailer operation ( $\mathrm{RO})$}

The RO strategy denotes that the e-retailer operates the physical showroom. In the RO strategy, the manufacturer's profit is $\pi_{m}^{r}=\left[\alpha\left(w-k q^{2}\right)+(1-\alpha)\left(s q-k q^{2}\right)\right] D$ and the e-retailer's profit is $\pi_{r}^{r}=\alpha(p-w) D-\frac{1}{2} h \mu^{2}$. Backward induction is used to solve the model. The equilibrium is shown in Lemma 4.6.

Lemma 4.6. In the RO strategy,

(1) when $k \leq \frac{(1-\alpha) s+\alpha}{2(1-\alpha) \alpha h}$, if $q \leq \frac{[(4 x-3) \alpha+(1-\alpha) s] h}{h k-2}, w^{r^{*}}=\frac{q^{2}+(2 x-1) \alpha h q}{\alpha h}, p^{r^{*}}=\frac{x \alpha h q+q^{2}}{\alpha h}$, and $\mu^{r^{*}}=\frac{q}{h}$; otherwise, $w^{r^{*}}=\frac{(2 x-1) \alpha q+q}{\alpha}, p^{r^{*}}=\frac{x \alpha q+q}{\alpha}, \mu^{r^{*}}=1$;

(2) when $k>\frac{(1-\alpha) s+\alpha}{2(1-\alpha) \alpha h}$, if $q \leq \frac{[(4 x-3) \alpha+(1-\alpha) s] h}{h k-2}, w^{r^{*}}=\frac{q^{2}+(2 x-1) \alpha h q}{\alpha h}, p^{r^{*}}=\frac{x \alpha h q+q^{2}}{\alpha h}$, and $\mu^{r^{*}}=\frac{q}{h}$; if $\frac{[(4 x-3) \alpha+(1-\alpha) s] h}{h k-2}<q \leq \frac{\alpha+(1-\alpha) s}{k}, w^{r^{*}}=\frac{\alpha q+k q^{2}-(1-\alpha) s q}{2 \alpha}, p^{r^{*}}=$ $\frac{(1-x) \alpha h k+(1-\alpha) s-\alpha] q^{2}+(1-\alpha) \alpha h[3 \alpha-(1-\alpha) s] q-k q^{3}}{2 \alpha[2(1-x) \alpha h-q]}$, and $\mu^{r^{*}}=\frac{\alpha q+(1-\alpha) s q-k q^{2}}{2[2(1-x) \alpha h-q]}$; otherwise, no product is produced.

Proof. In the RO strategy, the e-retailer's profit is $\pi_{r}^{r}=\alpha(p-w) D-\frac{1}{2} h \mu^{2}$. By solving $\frac{\partial \pi_{r}^{r}}{\partial p}=0$, the best responses of the e-retailer are obtained: (1) when $\mu<\frac{\alpha w-(2 x-1) \alpha q}{q}, p^{r^{*}}=\frac{(\alpha+\mu) q+\alpha w}{2 \alpha}, D^{r^{*}}=\frac{(\alpha+\mu) q-\alpha w}{2(1-x) \alpha q}$, and $\pi_{r}^{r^{*}}=\frac{[(\alpha+\mu) q-\alpha w]^{2}}{4(1-x) \alpha q}-\frac{1}{2} h \mu^{2} ;(2)$ when $\mu \geq \frac{\alpha w-(2 x-1) \alpha q}{q}, p^{r^{*}}=\frac{x \alpha q+\mu q}{\alpha}$, and $\pi_{r}^{r^{*}}=(x \alpha+\mu) q-\alpha w-\frac{1}{2} h \mu^{2}$.

(1) Case 1: $w<\frac{(2 x-1) \alpha q+q}{\alpha}(\mu<1)$.

When $\mu<\frac{\alpha w-(2 x-1) \alpha q}{q}, \mu^{r^{*}}=\frac{\alpha(q-w)}{2(1-x) \alpha h-q}$ if $w<q<\frac{w}{2 x-1}$. As $0<\mu^{r^{*}}<\mu<1$, we find that, if $\frac{q^{2}+(2 x-1) \alpha h q}{\alpha h}<w<\frac{(2 x-1) \alpha q+q}{\alpha}, \mu^{r^{*}}=\frac{\alpha(q-w)}{2(1-x) \alpha h-q}, p^{r^{*}}=\frac{\alpha h(1-x)(q+w)-w q}{2(1-x) \alpha h-q}$, and $D^{r^{*}}=\frac{\alpha h(q-w)}{2(1-x) \alpha h q-q^{2}}$; if $w \leq \frac{q^{2}+(2 x-1) \alpha h q}{\alpha h}, \mu^{r^{*}}=\frac{\alpha w-(2 x-1) \alpha q}{q}, p^{r^{*}}=(1-x) q+w$, and $D^{r^{*}}=1$. When $\mu \geq \frac{\alpha w-(2 x-1) \alpha q}{q}$, if $w \leq$ $\frac{q^{2}+(2 x-1) \alpha h q}{\alpha h}, \mu^{r^{*}}=\frac{q}{h}, p^{r^{*}}=\frac{q^{2}+\alpha x h q}{\alpha h}$, and $D^{r^{*}}=1$; if $\frac{q^{2}+(2 x-1) \alpha h q}{\alpha h}<w<\frac{(2 x-1) \alpha q+q}{\alpha}, \mu^{r^{*}}=\frac{\alpha w-(2 x-1) \alpha q}{q}$, $p^{r^{*}}=(1-x) q+w$, and $D^{r^{*}}=1$.

(2) Case 2: $w \geq \frac{(2 x-1) \alpha q+q}{\alpha}(\mu \geq 1)$.

When $\mu<\frac{\alpha w-(2 x-1) \alpha q}{q}, \mu^{r^{*}}=\frac{\alpha(q-w)}{2(1-x) \alpha h-q}$ if $w<q<\frac{w}{2 x-1}$. Considering $\mu^{r^{*}}<1$, we find that when $w>\frac{(1+\alpha) q-2(1-x) \alpha h}{\alpha}, \mu^{r^{*}}=\frac{\alpha(q-w)}{2(1-x) \alpha h-q}, p^{r^{*}}=\frac{\alpha h(1-x)(q+w)-w q}{2(1-x) \alpha h-q}$, and $D^{r^{*}}=\frac{\alpha h(q-w)}{2(1-x) \alpha h q-q^{2}}$. As $q<h$, we obtain that when $w \geq \frac{(2 x-1) \alpha q+q}{\alpha}, \mu^{r^{*}}=\frac{\alpha(q-w)}{2(1-x) \alpha h-q}, p^{r^{*}}=\frac{\alpha h(1-x)(q+w)-w q}{2(1-x) \alpha h-q}$, and $D^{r^{*}}=\frac{\alpha h(q-w)}{2(1-x) \alpha h q-q^{2}}$. When $\mu \geq \frac{\alpha w-(2 x-1) \alpha q}{q}, \mu^{r^{*}}=1, p^{r^{*}}=\frac{\alpha h(1-x)(q+w)-w q}{2(1-x) \alpha h-q}$, and $D^{r^{*}}=\frac{\alpha h(q-w)}{2(1-x) \alpha h q-q^{2}}$.

To sum up, (1) when $\mu<\frac{\alpha w-(2 x-1) \alpha q}{q}$; if $w \leq \frac{q^{2}+(2 x-1) \alpha h q}{\alpha h}, \mu^{r^{*}}=\frac{q}{h}, p^{r^{*}}=\frac{q^{2}+\alpha x h q}{\alpha h}$, and $D^{r^{*}}=1$; if $w>$ $\frac{q^{2}+(2 x-1) \alpha h q}{\alpha h}, \mu^{r^{*}}=\frac{\alpha(q-w)}{2(1-x) \alpha h-q}, p^{r^{*}}=\frac{\alpha h(1-x)(q+w)-w q}{2(1-x) \alpha h-q}$, and $D^{r^{*}}=\frac{\alpha h(q-w)}{2(1-x) \alpha h q-q^{2}}$. (2) When $\mu \geq \frac{\alpha w-(2 x-1) \alpha q}{q}$, if $w \leq \frac{q^{2}+(2 x-1) \alpha h q}{\alpha h}, \mu^{r^{*}}=\frac{q}{h}, p^{r^{*}}=\frac{q^{2}+\alpha x h q}{\alpha h}$, and $D^{r^{*}}=1$; if $\frac{q^{2}+(2 x-1) \alpha h q}{\alpha h}<w<\frac{(2 x-1) \alpha q+q}{\alpha} \mu^{r^{*}}=\frac{\alpha w-(2 x-1) \alpha q}{q}$, $p^{r^{*}}=(1-x) q+w$, and $D^{r^{*}}=1$; if $w \geq \frac{(2 x-1) \alpha q+q}{\alpha} \mu^{r^{*}}=1, p^{r^{*}}=\frac{\alpha h(1-x)(q+w)-w q}{2(1-x) \alpha h-q}$, and $D^{r^{*}}=\frac{\alpha h(q-w)}{2(1-x) \alpha h q-q^{2}}$.

Third, given the manufacturer's profit $\pi_{m}^{r}=\left[\alpha\left(w-k q^{2}\right)+(1-\alpha)\left(s q-k q^{2}\right)\right] D$, we firstly obtain $\frac{\partial^{2} \pi_{m}^{r}}{\partial w}=$ $\frac{-2 \alpha h}{2(1-x) \alpha h q-q^{2}}<0$ when $D^{r^{*}}=\frac{\alpha h(q-w)}{2(1-x) \alpha h q-q^{2}}$, which reveals that $\pi_{m}^{r}$ is a strictly concave function of $w$. When $D^{r^{*}}=1, \pi_{m}^{r}$ is an increasing function. Then we find the optimal wholesale price $w^{r^{*}}$. 
(1) When $\mu<\frac{\alpha w-(2 x-1) \alpha q}{q}$, if $w \leq \frac{q^{2}+(2 x-1) \alpha h q}{\alpha h}, w^{r^{*}}=\frac{q^{2}+(2 x-1) \alpha h q}{\alpha h}, \mu^{r^{*}}=\frac{q}{h}$, if $w>\frac{q^{2}+(2 x-1) \alpha h q}{\alpha h}$ $w^{r^{*}}=\frac{\alpha q+k q 2-(1-\alpha) s q}{2 \alpha}, \mu^{r^{*}}=\frac{\alpha q+(1-\alpha) s q-k q^{2}}{2[2(1-x) \alpha h-q]}$ only exist when $k>\frac{(1-\alpha) s+\alpha}{2(1-\alpha) \alpha h}$ and $q \leq \frac{\alpha+(1-\alpha) s}{k}$. As $\frac{\alpha q+(1-\alpha) s q-k q^{2}}{2[2(1-x) \alpha h-q]} \geq \frac{q}{h}$, we find that when $q \leq \frac{[(4 x-3) \alpha+(1-\alpha) s] h}{h k-2}, w^{r^{*}}=\frac{q^{2}+(2 x-1) \alpha h q}{\alpha h}, \mu^{r^{*}}=\frac{q}{h}$; when $\frac{[(4 x-3) \alpha+(1-\alpha) s] h}{h k-2}<q \leq \frac{\alpha+(1-\alpha) s}{k}, w^{r^{*}}=\frac{\alpha q+k q^{2}-(1-\alpha) s q}{2 \alpha}, \mu^{r^{*}}=\frac{\alpha q+(1-\alpha) s q-k q^{2}}{2[2(1-x) \alpha h-q]}$. Otherwise, $\mu^{r^{*}}=0$.

(2) When $\mu \geq \frac{\alpha w-(2 x-1) \alpha q}{q}$, if $w \leq \frac{q^{2}+(2 x-1) \alpha h q}{\alpha h}, w^{r^{*}}=\frac{q^{2}+(2 x-1) \alpha h q}{\alpha h}$, and $\mu^{r^{*}}=\frac{q}{h}$; if $\frac{q^{2}+(2 x-1) \alpha h q}{\alpha h}<w<$ $\frac{(2 x-1) \alpha q+q}{\alpha}, w^{r^{*}}=\frac{(2 x-1) \alpha q+q}{\alpha}$, and $\mu^{r^{*}}=1$; if $w \geq \frac{(2 x-1) \alpha q+q}{\alpha}, w^{r^{*}}=\frac{k q^{2}+(\alpha+1) q-(1-\alpha) s q}{2 \alpha}$, and $\mu^{r^{*}}=1$.

In conclusion, the optimal solutions are obtained. Proof ends.

Lemma 4.6 shows the equilibrium of the wholesale price and retail price in the RO strategy and illustrates the relationship between the optimal service decision and product quality. Compared with the CO strategy, it's worth noting that the optimal showroom service reaches the maximum value sharply when the product quality is high and the production cost coefficient is low. When the production cost coefficient is high, the RO strategy shows similar trends with the CO strategy. The optimal showroom service increases first and then decreases with product quality, as shown in Proposition 4.7.

Proposition 4.7. In the RO strategy, the optimal showroom service may decrease with the increase of product quality when the production cost coefficient is high. Formally, when $k>\frac{(1-\alpha) s+\alpha}{2(1-\alpha) \alpha h}$, if $\max \left\{\frac{[(4 x-3) \alpha+(1-\alpha) s] h}{h k-2}, \widehat{q}\right\}<q \leq \frac{\alpha+(1-\alpha) s}{k}, \frac{\partial \mu^{r^{*}}}{\partial q}<0$.

Proof. According to Lemma 4.6, when $k>\frac{(1-\alpha) s+\alpha}{2(1-\alpha) \alpha h}$, if $\frac{[(4 x-3) \alpha+(1-\alpha) s] h}{h k-2}<q \leq \frac{\alpha+(1-\alpha) s}{k}$, let $\frac{\partial \mu^{r^{*}}}{\partial q}=$ $\frac{k q^{2}+2(1-x) \alpha h[\alpha+(1-\alpha) s-2 k q]}{2[2(1-x) \alpha h-q]^{2}}=0$, and we obtain the equation $k q^{2}+2(1-x) \alpha h[\alpha+(1-\alpha) s-2 k q]=0$. We

find that this equation is the same as the equation in Proposition 4.2. So they has identical two roots: $\widehat{q}$ and $\bar{q}$ $(0<\widehat{q}<\breve{q})$. Therefore, when $\max \left\{\frac{[(4 x-3) \alpha+(1-\alpha) s] h}{h k-2}, \widehat{q}\right\}<q \leq \frac{\alpha+(1-\alpha) s}{k}, \mu^{r^{*}}=\frac{\alpha q+(1-\alpha) s q-k q^{2}}{2(1-x) \alpha h-q}$ is a monotonically decreasing function, where $\widehat{q}$ is the smaller solution of $k q^{2}+2(1-x) \alpha h[\alpha+(1-\alpha) s-2 k q]=0$. Proof ends.

According to Lemma 4.6 and Proposition 4.7, Figure 4 illustrates the trends of optimal showroom service in the RO strategy. When the production cost coefficient is high, the trends of the showroom service in the RO strategy are similar to that in the $\mathrm{CO}$ and RO strategies. However, when the production cost coefficient is low, the optimal showroom service reaches the maximum sharply with product quality. The reason is that the e-retailer does not need to undertake the salvage loss of return products according to the assumption of a full refund policy. Hence, the e-retailer tends to adopt the best showroom service to encourage consumer purchase behaviors to gain more marginal profit.

Comparing with the CO strategy, we find that the RO strategy may offer a better service than the CO strategy when the production cost coefficient is low, but the CO strategy is better when the production cost coefficient is high. Therefore, when production cost is low, the e-retailer should open the physical showroom separately than jointly. When production cost is high, the e-retailer should cooperate with the manufacturer.

\subsection{Equilibrium analysis}

\subsubsection{Service decision}

The service decisions in three strategies are discussed in this section, as illustrated in Figure 5. CO, MO, and $\mathrm{RO}$ represent the optimal showroom service in the $\mathrm{CO}, \mathrm{MO}$, and RO strategies, respectively. In order to analyze the showroom service comprehensively, the production cost coefficient is divided into two parts: the low $k$ case and the high $k$ case. We analyze the service decision from two perspectives of product quality and operation strategy in the following. 
(b)

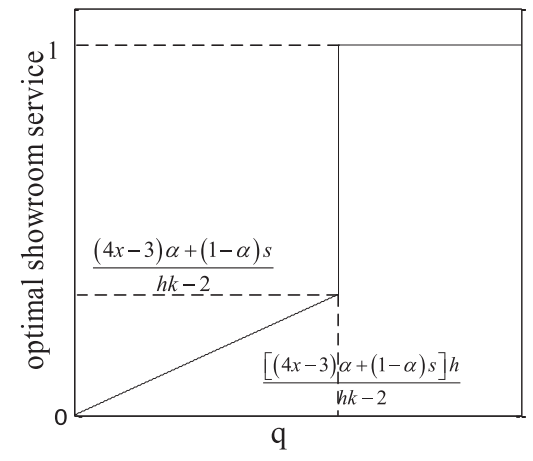

(b)

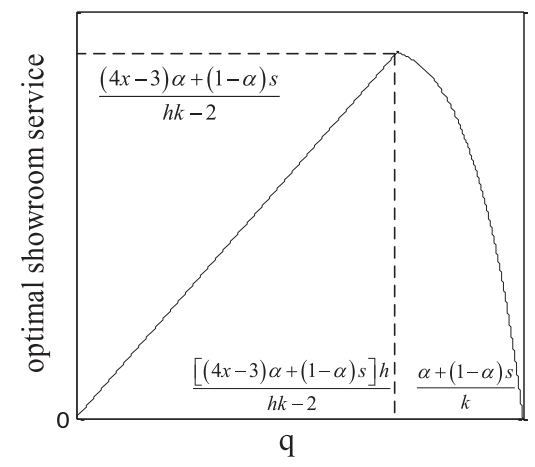

FiguRE 4. The optimal showroom service in the RO strategy. (a) When $k$ is low. (b) When $k$ is high.

(a)

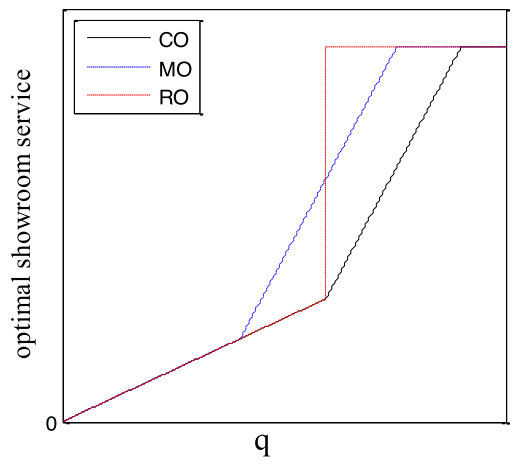

(b)

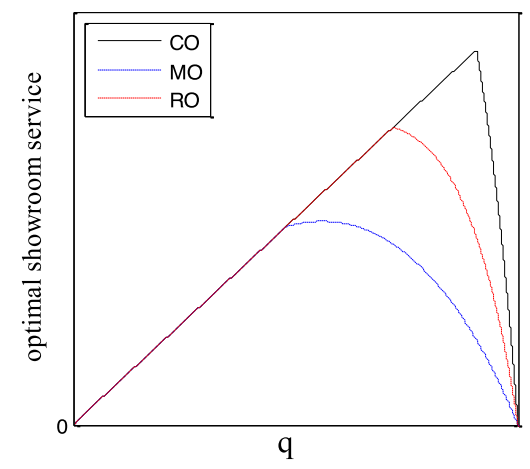

FIGURE 5. The optimal showroom service in the three strategies. (a) When $k$ is low. (b) When $k$ is high.

From the perspective of product quality, when product quality is low, the optimal showroom service is positively related to product quality and negatively relevant to showroom cost coefficient, and it is independent with the production cost coefficient. Meanwhile, the three strategies provide the same showroom service in this scenario, because the total profit in the whole market is higher than that in the partial market when product quality is low. Hence, the whole market is selected as the target market. However, when product quality is high, the showroom service trends rely on production cost. Comparing the optimal showroom services between the low and high $k$ cases, the summary is described in Corollary 4.8 .

Corollary 4.8. When the product quality is high, the high production cost coefficient restrains the showroom service in the three strategies.

Proof. In the CO strategy, when $\frac{[(2 x-1) \alpha+(1-\alpha) s] h}{h k-1}<q \leq \frac{\alpha+(1-\alpha) s}{k}, \mu^{c^{*}}=k q-(2 x-1) \alpha-(1-\alpha) s$ if $k \leq$ $\frac{(1-\alpha) s+\alpha}{2(1-\alpha) \alpha h} ; \mu^{c^{*}}=\frac{\alpha q+(1-\alpha) s q-k q^{2}}{2(1-x) \alpha h-q}$ if $k>\frac{(1-\alpha) s+\alpha}{2(1-\alpha) \alpha h}$. By solving $k q-(2 x-1) \alpha-(1-\alpha) s-\frac{\alpha q+(1-\alpha) s q-k q^{2}}{2(1-x) \alpha h-q}>$ 0 , we find that the optimal showroom service with the low production cost coefficient is higher than that with the high production cost coefficient. Also, the same findings are obtained in the MO and RO strategies. Proof ends. 
According to Corollary 4.8, we find that the optimal showroom service in the low $k$ case is better than that in the high $k$ case when product quality is high. In the low $k$ case, the manufacturer produces a high-quality product with a low production cost coefficient, such as luxury products. The enterprises are motivated to operate the physical showroom and encourage consumer purchase behaviors, thus expanding market coverage. However, in the high $k$ case, the optimal showroom service decreases with the high-quality product. The reason is that the promotion effect of showroom service decreases and the enterprises prefer to reduce the showroom service cost to maximize marginal profits.

Besides, from the perspective of operation strategy, we compared the showroom service decision among the three strategies. The conclusion is summarized in Corollary 4.9 .

Corollary 4.9. (1) When the production cost coefficient is low, the MO and the RO strategies may offer the better showroom service than the CO strategy. Formally, $\mu^{m^{*}} \geq \mu^{c^{*}}$ and $\mu^{r^{*}} \geq \mu^{c^{*}}$.

(2) When the production cost coefficient is high, the CO strategy may offer the better showroom service than the $M O$ and the RO strategies. Formally, $\mu^{c^{*}} \geq \mu^{r^{*}} \geq \mu^{m^{*}}$.

Proof. (1) When $k \leq \frac{(1-\alpha) s+\alpha}{4(1-\alpha) \alpha h}$, according to Corollary 4.5, we find that $\mu^{m^{*}} \geq \mu^{c^{*}}$. Then we compare $\mu^{c^{*}}$ and $\mu^{r^{*}}$. If $q \leq \frac{[(4 x-3) \alpha+(1-\alpha) s] h}{h k-2}, \mu^{c^{*}}=\mu^{r^{*}}=\frac{q}{h}$; If $q>\frac{[(4 x-3) \alpha+(1-\alpha) s] h}{h k-2}, \mu^{c^{*}} \leq \mu^{r^{*}}=1$. To sum up, $\mu^{m^{*}} \geq \mu^{c^{*}}$ and $\mu^{r^{*}} \geq \mu^{c^{*}}$.

(2) When $k>\frac{(1-\alpha) s+\alpha}{2(1-\alpha) \alpha h}$, if $q \leq \frac{[(4 x-3) \alpha+(1-\alpha) s] h}{h k-1}, \mu^{c^{*}}=\mu^{m^{*}}=\mu^{r^{*}}=\frac{q}{h}$; if $\frac{[(4 x-3) \alpha+(1-\alpha) s] h}{h k-1}<q \leq$ $\frac{[(4 x-3) \alpha+(1-\alpha) s] h}{h k-2}, \mu^{c^{*}}=\mu^{r^{*}}>\mu^{m^{*}} ;$ if $\frac{[(4 x-3) \alpha+(1-\alpha) s] h}{h k-2}<q \leq \frac{\alpha+(1-\alpha) s}{k}, \mu^{c^{*}}>\mu^{r^{*}}>\mu^{m^{*}}$.

To sum up, $\mu^{c^{*}} \geq \mu^{r^{*}} \geq \mu^{m^{*}}$. Proof ends.

Corollary 4.9 compares the optimal showroom service among the three strategies. According to the conventional intuition, the decentralized channel cannot provide better service than the centralized channel because of the double marginal effect. However, we find that the decentralized channel (the MO and RO strategies) may offer a better showroom service when the production cost coefficient is low. In particular, when the product quality is medium, the MO and RO strategies may provide better showroom service. However, when the production cost coefficient is high, the CO strategy may coordinate the service cost-sharing between the manufacturer and the e-retailer, thus reducing the risks and improving marginal profits. Hence, the CO strategy shows more advantages than the MO and RO strategies with high production cost coefficient.

\subsubsection{Price decision}

In this section, we analyze the price decision to show the optimal pricing path in the three strategies. Figure 6 demonstrates the optimal wholesale price and Figure 7 illustrates the optimal retail price in the low and high $k$ cases, respectively.

According to Figures 6 and 7, the optimal wholesale prices and the optimal retail price increase along with the showroom service. Moreover, when the production cost coefficient is low, the optimal retail prices in the MO and RO strategies may become higher than that in the CO strategy with medium-quality products. Therefore, in the low $k$ case, the enterprise should focus more on medium product quality if he (she) operates the physical showroom independently.

Nevertheless, the results are different in the high $k$ case. The MO and RO strategies may provide a lower retail price than the CO strategy when the production cost coefficient is high. Therefore, enterprises in the chain should cooperate to open physical showrooms. Meanwhile, compared with the RO strategy, the MO strategy may be higher in the optimal wholesale price, but lower in the optimal retail price. In the MO strategy, in view of total costs of production and showroom operation, the manufacturer prefers to raise the wholesale price and ensure his profit, so the optimal wholesale price in the MO strategy may be higher than that in the RO strategy. Similarly, the e-retailer prefers to increase the retail price to balance costs and benefits in the RO strategy. Thus, the RO strategy may offer a higher optimal retail price than that in the MO strategy. 
(a)

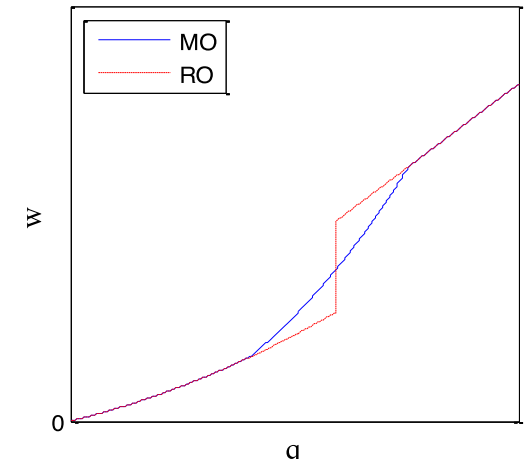

(b)

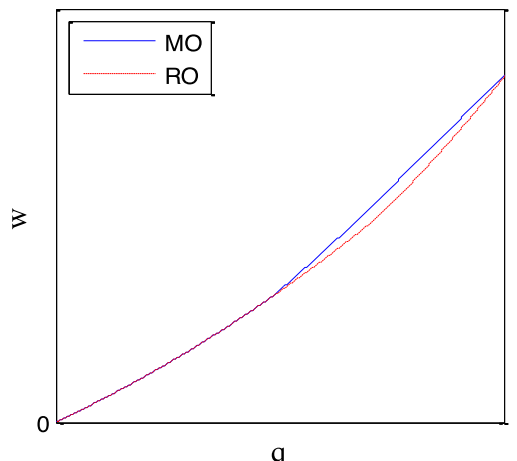

FiguRE 6. The optimal wholesale price in the three strategies. (a) When $k$ is low. (b) When $k$ is high.

(c)

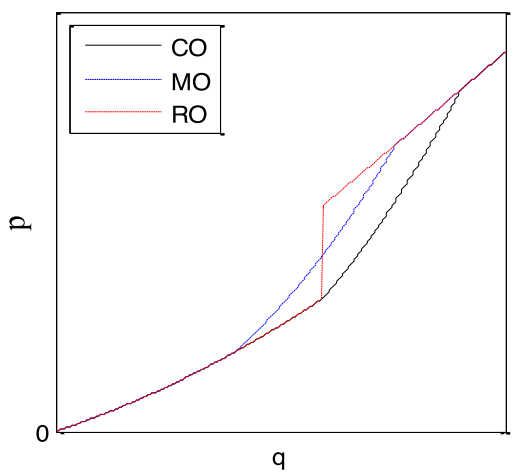

(d)

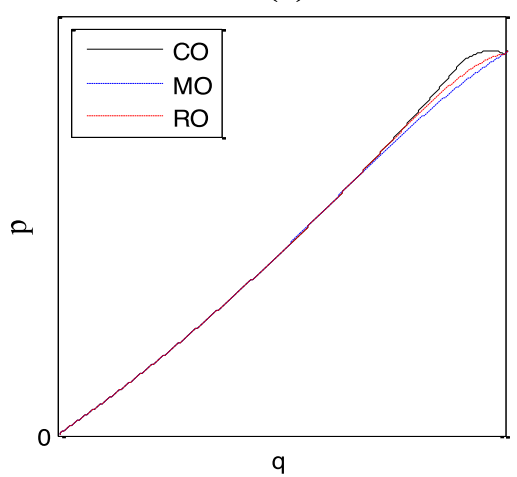

Figure 7 . The optimal retail price in the three strategies. (a) When $k$ is low. (b) When $k$ is high.

\subsubsection{Operation strategy}

In this section, we analyze the optimal showroom services in the $\mathrm{CO}, \mathrm{MO}$, and $\mathrm{RO}$ strategies. Comparing the three strategies, the optimal operation strategy is described in Proposition 4.10.

Proposition 4.10. When the production cost coefficient is low,

(1) if $\frac{[(4 x-3) \alpha+(1-\alpha) s] h}{h k-1}<q \leq \frac{[(4 x-3) \alpha+(1-\alpha) s] h}{h k-2}$, the MO strategy provides the optimal showroom service;

(2) if $\frac{[(4 x-3) \alpha+(1-\alpha) s] h}{h k-2}<q \leq \frac{(4 x-3) \alpha+(1-\alpha) s+1}{k}$, the RO strategy provides the optimal showroom service;

(3) if $\frac{(4 x-3) \alpha+(1-\alpha) s+1}{k}<q \leq \frac{(2 x-1) \alpha+(1-\alpha) s+1}{k}$, the RO and MO strategies provide the optimal showroom service;

(4) otherwise, the three strategies provide the optimal showroom service.

When the production cost coefficient is high,

(1) if $q \leq \frac{[(4 x-3) \alpha+(1-\alpha) s] h}{h k-1}$, the three strategies provide the optimal showroom service;

(2) if $\frac{[(4 x-3) \alpha+(1-\alpha) s] h}{h k-1}<q \leq \frac{[(4 x-3) \alpha+(1-\alpha) s] h}{h k-2}$, the CO and RO strategies provide the optimal showroom service;

(3) if $\frac{[(4 x-3) \alpha+(1-\alpha) s] h}{h k-2}<q \leq \frac{\alpha+(1-\alpha) s}{k}$, the CO strategy provides the optimal showroom service. 
(a)

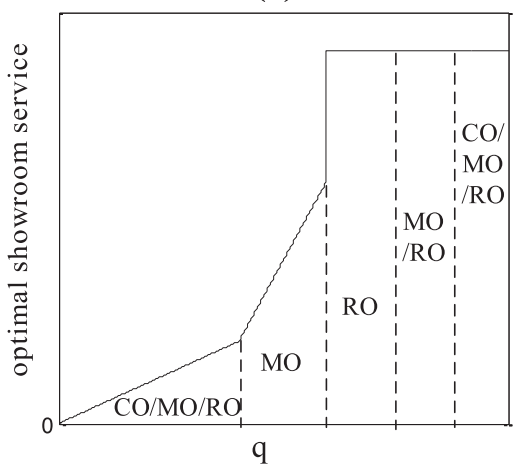

(b)

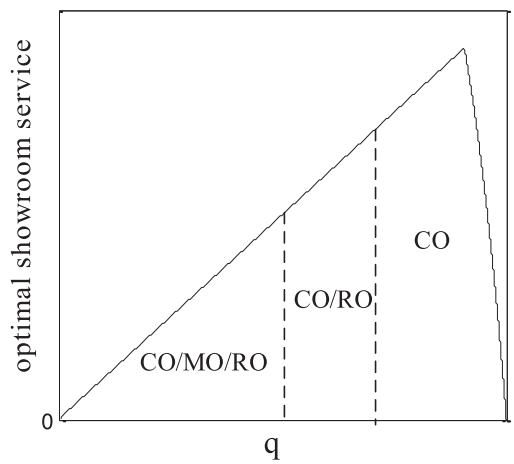

Figure 8. The optimal operation strategy based on product quality. (a) When $k$ is low. (b) When $k$ is high.

Proof. According to Lemmas 4.1, 4.3 and 4.6, when the production cost coefficient is low, we find that if $q \leq$ $\frac{[(4 x-3) \alpha+(1-\alpha) s] h}{h k-1}, \mu^{c^{*}}=\mu^{m^{*}}=\mu^{r^{*}}=\frac{q}{h}$, which means that the three strategies provide the optimal showroom service. If $\frac{[(4 x-3) \alpha+(1-\alpha) s] h}{h k-1}<q \leq \frac{[(4 x-3) \alpha+(1-\alpha) s] h}{h k-2}, \mu^{m^{*}}>\mu^{r^{*}}>\mu^{c^{*}}$, which means that the MO strategy provides the optimal showroom service. If $\frac{[(4 x-3) \alpha+(1-\alpha) s] h}{h k-2}<q \leq \frac{(4 x-3) \alpha+(1-\alpha) s+1}{k}, \mu^{r^{*}}>\mu^{m^{*}}>\mu^{c^{*}}$, which means that the RO strategy provides the optimal showroom service. If $\frac{(4 x-3) \alpha+(1-\alpha) s+1}{k}<q \leq \frac{(2 x-1) \alpha+(1-\alpha) s+1}{k}$, $\mu^{r^{*}}=\mu^{m^{*}}=1>\mu^{c^{*}}$, which means that the RO and MO strategies provide the optimal showroom service. Otherwise, $\mu^{r^{*}}=\mu^{m^{*}}=\mu^{c^{*}}=1$, revealing that the three strategies provide the same optimal showroom service.

When the production cost coefficient is high, if $q \leq \frac{[(4 x-3) \alpha+(1-\alpha) s] h}{h k-1}, \mu^{c^{*}}=\mu^{m^{*}}=\mu^{r^{*}}=\frac{q}{h}$, which means that the three strategies provide the same optimal showroom service. If $\frac{[(4 x-3) \alpha+(1-\alpha) s] h}{h k-1}<q \leq \frac{[(4 x-3) \alpha+(1-\alpha) s] h}{h k-2}$, $\mu^{c^{*}}=\mu^{r^{*}}>\mu^{m^{*}}$, which means that the $\mathrm{CO}$ and $\mathrm{RO}$ strategies provide the optimal showroom service. If $\frac{[(4 x-3) \alpha+(1-\alpha) s] h}{h k-2}<q \leq \frac{\alpha+(1-\alpha) s}{k}, \mu^{c^{*}}>\mu^{r^{*}}>\mu^{m^{*}}$, which means that the CO strategy provides the optimal showroom service. Prood ends.

Figure 8 visualizes Proposition 4.10, which illustrates the optimal operation strategy of physcial showrooms considering both product quality and production cost coefficient. Owing to the low production cost, the manufacturer and the e-retailer are more motivated to open the physical showroom to expand market demand and boost their margin profits, especially for medium-quality products. However, when the production cost coefficient is high, the CO strategy is optimal within all ranges of product quality. The summary is described in Corollary 4.11 .

Corollary 4.11. When the production cost coefficient is low, the MO strategy is optimal if $\frac{[(4 x-3) \alpha+(1-\alpha) s] h}{h k-1}<$ $q \leq \frac{[(4 x-3) \alpha+(1-\alpha) s] h}{h k-2}$, and the RO strategy is optimal if $\frac{[(4 x-3) \alpha+(1-\alpha) s] h}{h k-2}<q \leq \frac{(4 x-3) \alpha+(1-\alpha) s+1}{k}$. However, when the production cost coefficient is high, the CO strategy is optimal within all ranges of product quality.

Proof. According to Proposition 4.10, when the production cost coefficient is low, if $\frac{[(4 x-3) \alpha+(1-\alpha) s] h}{h k-1}<q \leq$ $\frac{[(4 x-3) \alpha+(1-\alpha) s] h}{h k-2}, \mu^{m^{*}}>\mu^{r^{*}}>\mu^{c^{*}}$, which means that only the MO strategy provides the optimal showroom service. If $\frac{[(4 x-3) \alpha+(1-\alpha) s] h}{h k-2}<q \leq \frac{(4 x-3) \alpha+(1-\alpha) s+1}{k}, \mu^{r^{*}}>\mu^{m^{*}}>\mu^{c^{*}}$, which means that only the RO strategy provides the optimal showroom service. When the production cost coefficient is high, $\mu^{c^{*}} \geq \mu^{r^{*}} \geq \mu^{m^{*}}$, which means that the $\mathrm{CO}$ strategy is optimal within all ranges of product quality. Proof ends.

Based on Corollary 4.11, when the production cost coefficient is high, the CO strategy provides the optimal showroom service within all ranges of product quality because of the double marginal effect, which is consistent 


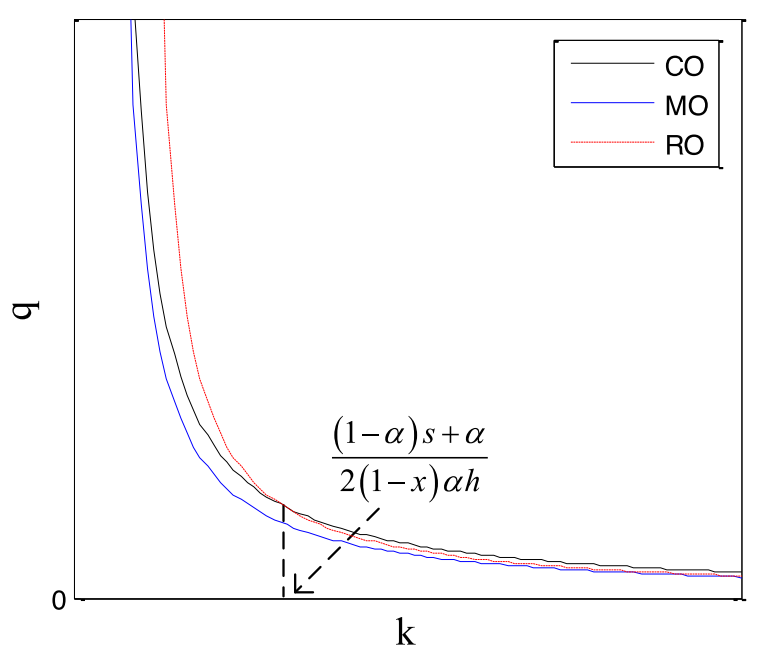

FiguRE 9. The optimal quality based on production cost coefficient.

with the traditional result. However, when the production cost coefficient is low, we find that the MO and RO strategies may provide a better service than the CO strategy, especially when product quality is mediate. Therefore, we conclude that the enterprise should open the physical showroom separately than jointly when the production cost coefficient is low, and simultaneously pay more attention to medium-quality products. When the production cost coefficient is high, the enterprises in the supply chain should cooperate to balance costs and benefits.

\section{Endogenous QUALITY}

To solve the above model, we recall Lemmas 4.1, 4.3, and 4.6 to analyze the optimal product quality and service decision in three strategies. According to the equilibrium, we firstly confirm the model's robustness. When product quality is endogenous, all the conclusions in this paper are valid. Further, we study the relationship between product quality and operation strategy. Previous works show that the CO strategy offers higher product quality than the $\mathrm{MO}$ and $\mathrm{RO}$ strategies owing to the double marginal effect. In contrast to the above conclusion, we find that product quality may be higher in the RO strategy when the production cost coefficient is moderate, as illustrated in Corollary 5.1.

Corollary 5.1. When the production cost coefficient is moderate, the RO strategy may provide a higher product quality than the CO strategy. Formally, when $\frac{2}{h}<k<\frac{(1-\alpha) s+\alpha}{2(1-x) \alpha h}, q^{r^{*}}>q^{c^{*}}$.

Proof. According to Lemma 4.1, when $q \leq \frac{[(2 x-1) \alpha+(1-\alpha) s] h}{h k-1}, \mu^{c^{*}}=\frac{q}{h}$. By optimizing the manufacturer's profit $\pi_{m}^{c^{*}}=\frac{1}{2}\left[(x \alpha+\mu) q+(1-\alpha) s q-k q^{2}-\frac{1}{2} h \mu^{2}\right]$, we first obtain $\frac{\partial^{2} \pi_{m}^{c^{*}}}{\partial q^{2}}<0$ and then solve $\frac{\partial \pi_{m}^{c^{*}}}{\partial q}=0$ to find that when $q \leq \frac{[(2 x-1) \alpha+(1-\alpha) s] h}{h k-1}, q^{c^{*}}=\frac{[x \alpha+(1-\alpha) s] h}{2 k h-1}$, and $\mu^{c^{*}}=\frac{q}{h}$.

Similarly, we get the optimal product quality $q^{r^{*}}$ from Lemma 4.6. By optimizing the manufacturer's profit $\pi_{m}^{r}=\alpha\left(w-k q^{2}\right)+(1-\alpha)\left(s q-k q^{2}\right)$, we firstly obtain $\frac{\partial^{2} \pi_{m}^{r^{*}}}{\partial q^{2}}<0$ when $k>\frac{2}{h}$ and then solve $\frac{\partial \pi_{m}^{r^{*}}}{\partial q}=0$ to find that when $q \leq \frac{[(4 x-3) \alpha+(1-\alpha) s] h}{h k-2}, q^{r^{*}}=\frac{[(2 x-1) \alpha+(1-\alpha) s] h}{2 k h-2}$, and $\mu^{r^{*}}=\frac{q}{h}$. Comparing the above two cases, we find that when $\frac{2}{h}<k<\frac{(1-\alpha) s+\alpha}{2(1-x) \alpha h}, q^{r^{*}}-q^{c^{*}}=\frac{[(2 x-1) \alpha+(1-\alpha) s] h}{2 k h-2}-\frac{[x \alpha+(1-\alpha) s] h}{2 k h-1}>0$. Prood ends.

Figure 9 visualizes Corollary 5.1 and shows the relationship between the optimal product quality and production cost coefficient. Comparing the optimal product quality in the three strategies, the CO strategy provides 
optimal product quality among the three strategies when the production cost coefficient is relatively high. This result is consistent with the previous work $[26,36]$. However, when the production cost coefficient is moderate, the RO strategy may provide higher product quality than the CO strategy. In this case, unlike the exogenous product scenario, the endogenous quality allows the manufacturer to choose high-quality products to induce the e-retailer to offer high showroom service to consumers. Therefore, when the e-retailer provides the showroom service, the decentralized channel may produce a higher product quality than the centralized channel.

\section{EXTENSION}

To further verify the model's robustness, Section 6.1 discusses the high consumer heterogeneity. Then, the impact of a partial refund policy is analyzed in Section 6.2.

\subsection{Consumer heterogeneity}

In this section, we discuss the scenario of high consumer heterogeneity, namely, $x(x=0)$. According to Lemmas 4.1, 4.3, and 4.6, the results in MO strategy are based on the lower consumer heterogeneity $x\left(x>\frac{3}{4}\right)$, comparing with the assumption of $x>\frac{1}{2}$ in the RO strategy. As a consequence, the MO strategy is selected to analyze the assumption of high consumer heterogeneity.

We recall the Lemmas 4.1 and 4.3. When consumer heterogeneity is high, only the partial market is covered. Then we plug the $x=0$ into Lemmas 4.1 and 4.3, and obtain the optimal showroom service. The results are described in the following. In the CO strategy, when $q \leq \frac{\alpha+(1-\alpha) s}{k}, \mu^{c^{*}}=\frac{\alpha q+(1-\alpha) s q-k q^{2}}{2 \alpha h-q}$; Otherwise, $\mu^{c^{*}}=0$. In the MO strategy, when $q \leq \frac{\alpha+(1-\alpha) s}{k}, \mu^{m^{*}}=\frac{\alpha q+(1-\alpha) s q-k q^{2}}{4 \alpha h-q}$; Otherwise, $\mu^{m^{*}}=0$. Therefore, it is concluded that when consumer heterogeneity is high, the CO strategy also offers a better showroom service than the RO strategy.

When product quality is relatively low, the optimal showroom service increases; When the product quality is relatively high, consumers are more sensitive to the product price, so enterprises tend to lower both the showroom service and price. As a result, the optimal showroom service decreases with product quality. Owing to the double marginal effect, the optimal showroom service in the decentralized channel is lower than that in the centralized channel. Consequently, when consumer heterogeneity is high, the conclusion of optimal showroom service is invariable.

\subsection{Refund policy}

In the model, we assume that the consumer's return cost is 0 . However, in reality, some e-retailers may provide a partial refund for returned products. To capture this scenario, we extend our model to consider a partial refund policy. Let $r$ stand for the refund and the consumer's utility is described as: $U(\theta)=\alpha(\theta q-p)+(1-\alpha)(r-p)+$ $\mu q$. To simplify the above function, the consumer's utility function is rewritten as $U(\theta)=\alpha \theta q-p+(1-\alpha) r+\mu q$. Given the assumption of $x>\frac{3}{4}$, the demand function is $D=1-\frac{p-\mu q-(1-\alpha) r}{\alpha q}$.

In the CO strategy, the profit of the supply chain is $\pi_{s c}^{c}=\left[\alpha\left(p-k q^{2}\right)+(1-\alpha)\left(p-r+s q-k q^{2}\right)\right] D-\frac{1}{2} h \mu^{2}$. By solving $\frac{\partial \pi_{s c}^{c}}{\partial p}=0$, the optimal retail price is $p^{c^{*}}=\frac{k q^{2}+(\alpha+\mu) q-(1-\alpha) q}{2 \alpha}+(1-\alpha) r$. Comparing with the optimal retail price under the full refund, we find that the optimal retail price under the partial refund policy is lower. Meanwhile, the negative impact of the partial refund policy on consumers is offset by the positive impact of lowering the retail price. That is to say, if the enterprises adopt the partial refund policy, the negative impact of the partial refund is also offset by appropriately lowering the retail price in the CO strategy. Therefore, the supply chain may obtain the same profit by selecting a corresponding retail price. In the MO strategy, the profit of the retailer is $\pi_{r}^{m}=[\alpha(p-w)+(1-\alpha)(p-r)] D$; In the RO strategy, the profit of the e-retailer is $\pi_{r}^{r}=$ $[\alpha(p-w)+(1-\alpha)(p-r)] D-\frac{1}{2} h \mu^{2}$. By solving above models, we find that whether the manufacturer or the e-retailer provides the showroom service, the optimal retail prices are same as $p^{m^{*}}=p^{r^{*}}=\frac{(\alpha+\mu) q+w}{2}+(1-\alpha) r$. 
As a result, we find that whether the assumption is a full refund policy or a partial refund policy, the results of our model are valid because the disadvantages of the partial refund policy are offset by the advantages of lowering the retail price. Consequently, when a partial refund policy is introduced, if the refund is high, the e-retailer should adopt a relatively high retail price; Otherwise, a lower retail price may be a better option.

\section{Conclusion}

\subsection{Theoretical implications}

The convenience of information acquisition enables consumers to switch their purchase channels in a sophisticated manner. To keep up with the diverse needs of consumers, the physical showroom is introduced to improve the consumer experience. In this paper, we study the $\mathrm{CO}, \mathrm{MO}$, and $\mathrm{RO}$ strategies of the physical showroom under e-commerce. Considering the production cost coefficient, we analyze the joint decision of the showroom service and pricing based on product quality in the three operation strategies. Here are some interesting conclusions.

First, conventional wisdom suggests that the high-quality product should be offered a high service than the low-quality product. Interestingly, we find that the optimal showroom service may descend with the increase of product quality when the production cost coefficient is high. Besides, when the product quality is high, we find that the optimal showroom service decision with the low production cost coefficient is higher than the high production cost coefficient. In other words, the high production cost coefficient restrains the improvement of optimal showroom service.

Second, the optimal operation strategy is analyzed in this paper. Our result reveals that enterprises should consider both product quality and production cost before making the showroom service decision. When the production cost coefficient is high, the MO and RO strategies may offer a better showroom service than the CO strategy. However, when the production cost coefficient is high, the CO strategy offers a better showroom service than the MO and RO strategies. Besides, medium-quality products should receive more attention regardless of the production cost coefficient.

Third, in the scenario of endogenous product, the RO strategy may provide higher product quality than the $\mathrm{CO}$ strategy when the production cost coefficient is moderate. The endogenous quality allows the manufacturer to choose high-quality products to induce the e-retailer to offer high showroom service to consumers, thus optimizing their profits. Therefore, when the e-retailer provides the showroom service, the decentralized channel may produce higher product quality than the centralized channel.

\subsection{Managerial implications}

We hope that our results are valuable for firms adopting showroom strategies. There are two aspects of managerial implications. For the manufacturer, he should open the physical showroom separately with the low production cost coefficient. Although the manufacturer undertakes the operational cost of the physical showroom, the physical showroom not only improves his profit, but also can effectively enhance the premium capacity of brands, and thus enhance corporate reputation. When the production cost coefficient is high, the manufacturer should cooperate with the e-retailer to balance costs and benefits, which can effectively reduce the risk of assets to achieve a long-term cooperation strategy in the supply chain. Besides, we also analyze the scenario of endogenous product quality. As production cost management is crucial for the manufacturer, some specific measures are suggested to decrease cost and improve efficiency based on the concept of lean production, for example, standardized production, process optimization, and technical innovation.

As for the e-retailer, when the production cost coefficient is low, the optimal operation strategy is also to open the physical showroom independently. However, when the production cost coefficient is high, the optimal operation strategy for the e-retailer is to cooperate with the manufacturer. In addition, when the e-retailer provides showroom service, our results show that the manufacturer may produce higher product quality than the centralized channel. 


\subsection{Future research}

This article provides several directions for future research. First, manufacturers produce various products of different quality levels, and this paper only considers a single product in the market. Furthermore, we consider the competitive products. Second, with the exception of paying attention to product quality, consumer purchase decisions are also affected by some behavioral factors, such as reference effects and anticipated regret. Therefore, considering consumers' behavioral factors is also an important research direction of this paper. Third, future studies can verify the conclusions of this paper from the perspective of empirical research.

Acknowledgements. The authors would like to thank the anonymous reviewers and the editor for their insightful comments and suggestions. The authors acknowledge the financial support from the Project of the National Natural Science Foundation of China (71871150); the Sichuan University Innovation Project (2021CXC22).

\section{REFERENCES}

[1] V. Abhishek, K. Jerath and Z.J. Zhang, Agency selling or reselling? Channel structures in electronic retailing. Manage. Sci. 62 (2016) 2259-2280.

[2] N. Beck and D. Rygl, Categorization of multiple channel retailing in multi-, cross-, and omni-channel retailing for retailers and retailing. J. Retail. Consum. Serv. 27 (2015) 170-178.

[3] D. Bell, S. Gallino and A. Moreno, Showrooms and information provision in omni-channel retail. Prod. Oper. Manag. 24 (2015) 360-362.

[4] D.R. Bell, S. Gallino and A. Moreno, Offline showrooms in omnichannel retail: demand and operational benefits. Manage. Sci. 64 (2018) 1629-1651.

[5] E. Biyalogorsky and O. Koenigsberg, The design and introduction of product lines when consumer valuations are uncertain. Prod. Oper. Manag. 23 (2014) 1539-1548.

[6] E. Brynjolfsson, Y.J. Hu and M.S. Rahman, Competing in the age of omnichannel retailing. Mit. Sloan. Manage. Rev. 54 (2013) 23-29.

[7] F. Caro, A.G. Kök and V. Martínez-de-Albéniz, The future of retail operations. Manuf. Serv. Oper. Manag. 22 (2020) 47-58.

[8] K. Cattani, W. Gilland, H.S. Heese and J. Swaminathan, Boiling frogs: pricing strategies for a manufacturer adding a direct channel that competes with the traditional channel. Prod. Oper. Manag. 15 (2006) 40-56.

[9] X. Chen, X. Wang and X. Jiang, The impact of power structure on the retail service supply chain with an O2O mixed channel. J. Oper. Res. Soc. 67 (2016) 294-301.

[10] J. Chen, L. Liang, D.-Q. Yao and S. Sun, Price and quality decisions in dual-channel supply chains. Eur. J. Oper. Res. 259 (2017) 935-948.

[11] J. Chen, W. Zhang and Z. Liu, Joint pricing, services and quality decisions in a dual-channel supply chain. RAIRO-Oper. Res. 54 (2020) 1041-1056.

[12] W.Y.K. Chiang, D. Chhajed and J.D. Hess, Direct-marketing, indirect profits: a strategic analysis of dual-channel supply-chain design. Manage. Sci. 49 (2003) 1-20.

[13] S. Du, L. Wang and L. Hu, Omnichannel management with consumer disappointment aversion. Int. J. Prod. Econ. 215 (2019) $84-101$.

[14] X. Fan, J. Wang and T. Zhang, For showing only, or for selling? The optimal physical store mode selection decision for e-tailers under competition. Int. Trans. Oper. Res. 28 (2019) 764-783.

[15] M. Fassnacht, S.E. Beatty and M. Szajna, Combating the negative effects of showrooming: successful salesperson tactics for converting showroomers into buyers. J. Bus. Res. 102 (2019) 131-139.

[16] N.V. Fernández, M.J.S. Pérez and R. Vázquez-Casielles, Webroomers versus showroomers: are they the same? J. Bus. Res. 92 (2018) 300-320.

[17] X. Fu, S. Liu, W. Shen and G. Han, Managing strategies of product quality and price based on trust under different power structures. RAIRO-Oper. Res. 55 (2021) 701-725.

[18] S. Gallino, A. Moreno and I. Stamatopoulos, Channel integration, sales dispersion, and inventory management. Manage. Sci. 63 (2017) 2813-2831.

[19] F. Gao and X. Su, Online and offline information for omnichannel retailing. Manuf. Serv. Oper. Manag. 19 (2017) 84-98.

[20] F. Gao and X. Su, Omnichannel service operations with online and offline self-order technologies. Manage. Sci. 64 (2018) 3595-3608.

[21] S. Gensler, S.A. Neslin and P.C. Verhoef, The showrooming phenomenon: it's more than just about price. J. Interact. Mark. 38 (2017) 29-43.

[22] B.C. Giri, C. Mondal and T. Maiti, Optimal product quality and pricing strategy for a two-period closed-loop supply chain with retailer variable markup. RAIRO-Oper. Res. 53 (2019) 609-626.

[23] X. Guan, Y. Wang, Z. Yi and Y.J. Chen, Inducing consumer online reviews via disclosure. Prod. Oper. Manag. 29 (2020) 1956-1971. 
[24] A. Ha, X. Long and J. Nasiry, Quality in supply chain encroachment. Manuf. Serv. Oper. Manag. 18 (2016) $280-298$.

[25] L. Hsiao and Y.-J. Chen, Strategic motive for introducing internet channels in a supply chain. Prod. Oper. Manag. 23 (2014) $36-47$.

[26] A.P. Jeuland and S.M. Shugan, Managing channel profits. Marketing Sci. 27 (2008) 52-69.

[27] G. Li, T. Zhang and G.K. Tayi, Inroad into omni-channel retailing: physical showroom deployment of an online retailer. Eur. J. Oper. Res. 283 (2020) 676-691.

[28] B. Ma, C. Di and L. Hsiao, Return window decision in a distribution channel. Prod. Oper. Manag. 29 (2020) $2121-2137$.

[29] A. Mehra, S. Kumar and J.S. Raju, Competitive strategies for brick-and-mortar stores to counter "Showrooming". Manage. Sci. 64 (2018) 3076-3090.

[30] U. Narang and V. Shankar, Mobile app introduction and online and offline purchases and product returns. Marketing Sci. 38 (2019) 756-772.

[31] E. Ofek, Z. Katona and M. Sarvary, "Bricks and Clicks": the impact of product returns on the strategies of multichannel retailers. Marketing Sci. 30 (2011) 42-60.

[32] D. Rigby, The future of shopping. Harvard Bus. Rev. 89 (2011) 64-76.

[33] H. Shi, Y. Liu and N.C. Petruzzi, Consumer heterogeneity, product quality, and distribution channels. Manage. Sci. 59 (2013) $1162-1176$.

[34] J.D. Shulman, A.T. Coughlan and R.C. Savaskan, Optimal reverse channel structure for consumer product returns. Marketing Sci. 29 (2010) 1071-1085.

[35] P.C. Verhoef, P.K. Kannan and J.J. Inman, From multi-channel retailing to omni-channel retailing introduction to the special issue on multi-channel retailing. J. Retailing. 91 (2015) 174-181.

[36] J.M. Villas-Boas, Product line design for a distribution channel. Marketing Sci. 17 (1998) 156-169.

[37] X. Xu, Optimal price and product quality decisions in a distribution channel. Manage. Sci. 55 (2009) 1347-1352.

[38] M. Xue and J. Zhang, Supply chain encroachment with quality decision and different power structures. RAIRO-Oper. Res. 54 (2020) 693-718.

[39] D. Yang and X. Zhang, Quick response and omnichannel retail operations with the ship-to-store program. Int. Trans. Oper. Res. 27 (2020) 3007-3030.

[40] J. Zhang, Q. Xu and Y. He, Omnichannel retail operations with consumer returns and order cancellation. Transp. Res. E-Log. 118 (2018) 308-324.

[41] T. Zhang, G. Li, T.C.E. Cheng and S. Shum, Consumer inter-product showrooming and information service provision in an omni-channel supply chain. Decis. Sci. 51 (2020) 1232-1264.

\section{Subscribe to Open (S20)}

\section{A fair and sustainable open access model}

This journal is currently published in open access under a Subscribe-to-Open model (S2O). S2O is a transformative model that aims to move subscription journals to open access. Open access is the free, immediate, online availability of research articles combined with the rights to use these articles fully in the digital environment. We are thankful to our subscribers and sponsors for making it possible to publish this journal in open access, free of charge for authors.

\section{Please help to maintain this journal in open access!}

Check that your library subscribes to the journal, or make a personal donation to the $\mathrm{S} 2 \mathrm{O}$ programme, by contacting subscribers@edpsciences.org

More information, including a list of sponsors and a financial transparency report, available at: https://www. edpsciences.org/en/maths-s2o-programme 